DEMOGRAPHIC RESEARCH

VOLUME 29, ARTICLE 43, PAGES 1187-1226

PUBLISHED 10 DECEMBER 2013

http://www.demographic-research.org/Volumes/Vol29/43/

DOI: $\quad$ 10.4054/DemRes.2013.29.43

Research Material

Integrating uncertainty in time series population forecasts: An illustration using a simple projection model

Guy J. Abel

Jonathan J. Forster

Peter W.F. Smith
Jakub Bijak

James Raymer

Jackie S.T. Wong

(c) 2013 Abel, Bijak, Forster, Raymer, Smith \& Wong.

This open-access work is published under the terms of the Creative Commons Attribution NonCommercial License 2.0 Germany, which permits use, reproduction \& distribution in any medium for non-commercial purposes, provided the original author(s) and source are given credit. See http://creativecommons.org/licenses/by-nc/2.0/de/ 


\section{Table of Contents}

$1 \quad$ Introduction $\quad 1188$

2 Data 1189

$3 \quad$ Statistical background $\quad 1191$

3.1 Autoregression model 1191

3.2 Stochastic volatility model 1192

3.3 Random variance shift model 1192

3.4 Bayesian inference 1193

3.5 Model uncertainty 1194

$4 \quad$ Computation and forecasts 1195

4.1 Individual autoregressive models $\quad 1196$

$\begin{array}{ll}4.2 & \text { Individual stochastic volatility models } \\ & 1202\end{array}$

4.3 Individual random variance shift models $\quad 1206$

$\begin{array}{ll}4.4 & \text { Model-averaged forecasts } \\ \end{array}$

$5 \quad$ In-sample forecast validation $\quad 1213$

6 Conclusion $\quad 1215$

$7 \quad$ Acknowledgements 1216

$\begin{array}{ll}\text { References } & 1217\end{array}$

$\begin{array}{ll}\text { Appendix } & 1221\end{array}$ 


\title{
Integrating uncertainty in time series population forecasts: An illustration using a simple projection model
}

\author{
Guy J. Abel $^{1}$ \\ Jakub Bijak ${ }^{2}$ \\ Jonathan J. Forster ${ }^{2}$ \\ James Raymer ${ }^{3}$ \\ Peter W.F. Smith ${ }^{2}$ \\ Jackie S.T. Wong ${ }^{4}$
}

\begin{abstract}
BACKGROUND

Population forecasts are widely used for public policy purposes. Methods to quantify the uncertainty in forecasts tend to ignore model uncertainty and to be based on a single model.
\end{abstract}

\section{OBJECTIVE}

In this paper, we use Bayesian time series models to obtain future population estimates with associated measures of uncertainty. The models are compared based on Bayesian posterior model probabilities, which are then used to provide model-averaged forecasts.

\section{METHODS}

The focus is on a simple projection model with the historical data representing population change in England and Wales from 1841 to 2007. Bayesian forecasts to the year 2032 are obtained based on a range of models, including autoregression models, stochastic volatility models and random variance shift models. The computational steps to fit each of these models using the OpenBUGS software via R are illustrated.

\section{RESULTS}

We show that the Bayesian approach is adept in capturing multiple sources of uncertainty

\footnotetext{
${ }^{1}$ Corresponding author: Wittgenstein Centre (IIASA, VID/ÖAW, WU), Vienna Institute of Demography/Austrian Academy of Sciences, Austria. E-mail: guy.abel@ oeaw.ac.at.

${ }^{2}$ ESRC Research Centre for Population Change, University of Southampton, United Kingdom.

${ }^{3}$ Australian Demographic and Social Research Institute, Australian National University, Australia.

${ }^{4}$ Mathematical Sciences, University of Southampton, United Kingdom.
} 
in population projections, including model uncertainty. The inclusion of non-constant variance improves the fit of the models and provides more realistic predictive uncertainty levels. The forecasting methodology is assessed through fitting the models to various truncated data series.

\section{Introduction}

In this paper, a detailed exposition of the Bayesian approach to time series forecasts of population totals is provided. The aim is to encourage the use of Bayesian methods by researchers interested in population forecasting. The main motivation behind this work is the need to incorporate the assessment of uncertainty into population forecasts (Alho and Spencer 1985; Keyfitz 1991; Lee 1998). The rationale for considering the Bayesian approach is that it offers a more natural framework than traditional frequentist methods to forecast future population with uncertainty. First, variability in the data and uncertainties in the parameters and model choice are explicitly included using probability distributions. Second, the predictive distributions follow directly from the probabilistic model applied. Third, it allows the incorporation of expert judgements, including their uncertainty, into the model framework. As a result, probabilistic population forecasts, with more reliable and coherent estimates of predictive uncertainty, can be obtained for a particular projection model.

The paper also includes an annotated implementation of Bayesian time series models in the dedicated software, OpenBUGS (Lunn et al. 2009), implemented via the R statistical package. To illustrate the Bayesian approach to population forecasting, we focus on the simplest case, a single time series of population change described in the next section. In Section 3, we set out the notation and describe the models used in this study. These include autoregression models for time series data, stochastic volatility models and models with variance shifts to account for heterogeneity. We also outline the Bayesian methods used for parameter estimation and for model averaging. In Section 4, we demonstrate how to estimate model parameters in each of the time series models using the OpenBUGS software via R. The fully probabilistic population forecasts from 2008 to 2032 from these models are also presented. In Section 5, we illustrate the performance of our forecasting methodology by fitting the models to various truncated data series and by comparing predictive distributions against actual observed data, outside the fitting period. Finally, we end the paper with a summary and some suggestions for extending the proposed approach. 


\section{Data}

A historical series of the England and Wales population totals are used to introduce the Bayesian approach to time series forecasting. These data were obtained from $\mathrm{The} \mathrm{Hu}-$ man Mortality Database ${ }^{4}$. The mid-year population totals from 1841 to 2007, including military personnel, are presented in the top panel of Figure 1. Here, we see that the population totals in England and Wales exhibited a steady increase over time, rising from 15.8 million in 1841 to 53.9 million in 2007. Brief periods of slight population decline are visible during the First World War and the 1918 influenza pandemic. Also noticeable is a period of levelling off in the population totals during the 1970s and 1980s, a result of net emigration and a lower rate of natural increase.

The features of population change are more evident when the annual rates of growth, plotted in the second panel of Figure 1, are considered. Detailed explanations for these patterns can be found in various books on British population history (Wrigley and Schofield 1989; Coleman and Salt 1992; Anderson 1996; Hinde 2003). The following provides a very brief account. The population growth rates were highest during the 1840-1910 period. This was predominantly due to the decrease in mortality occurring before the decline in fertility, which remained at pre-industrial levels for much of this period. Between the two World Wars, the rate of growth remained low in comparison with the later half of the 19th century and early 20th century. This was driven by the effects of low fertility from economic depression and a change in sociological factors. After the Second World War, population growth rates increased initially, through a short-lived fertility rise associated with demobilization, followed by a more substantial increase (baby boom) in fertility during the 1950s and early 1960s. In the late 1970s and early 1980s, the levels of population growth slowed down before rising in more recent decades though net immigration and higher fertility levels.

A time series of historical data is used to forecast the future rates of population growth. We are primarily interested in identifying the time series models that best fit these data in order ultimately to specify probabilistic intervals in forecasted populations. As we can see from Figure 1, the annual rates of growth have varied considerably over time. The models, described in the next section, provide alternative probabilistic specifications for this variation, and hence can be used to predict future variations in population change. These models are sufficiently flexible to describe a long series, and provide realistic projections, without the need to choose a strategic starting point for the data series to best reflect future variation.

\footnotetext{
${ }^{4}$ Data available from http://www.mortality.org
} 
Abel et al:: Integrating uncertainty in time series population forecasts

Figure 1: $\quad$ England and Wales population data, 1841-2007
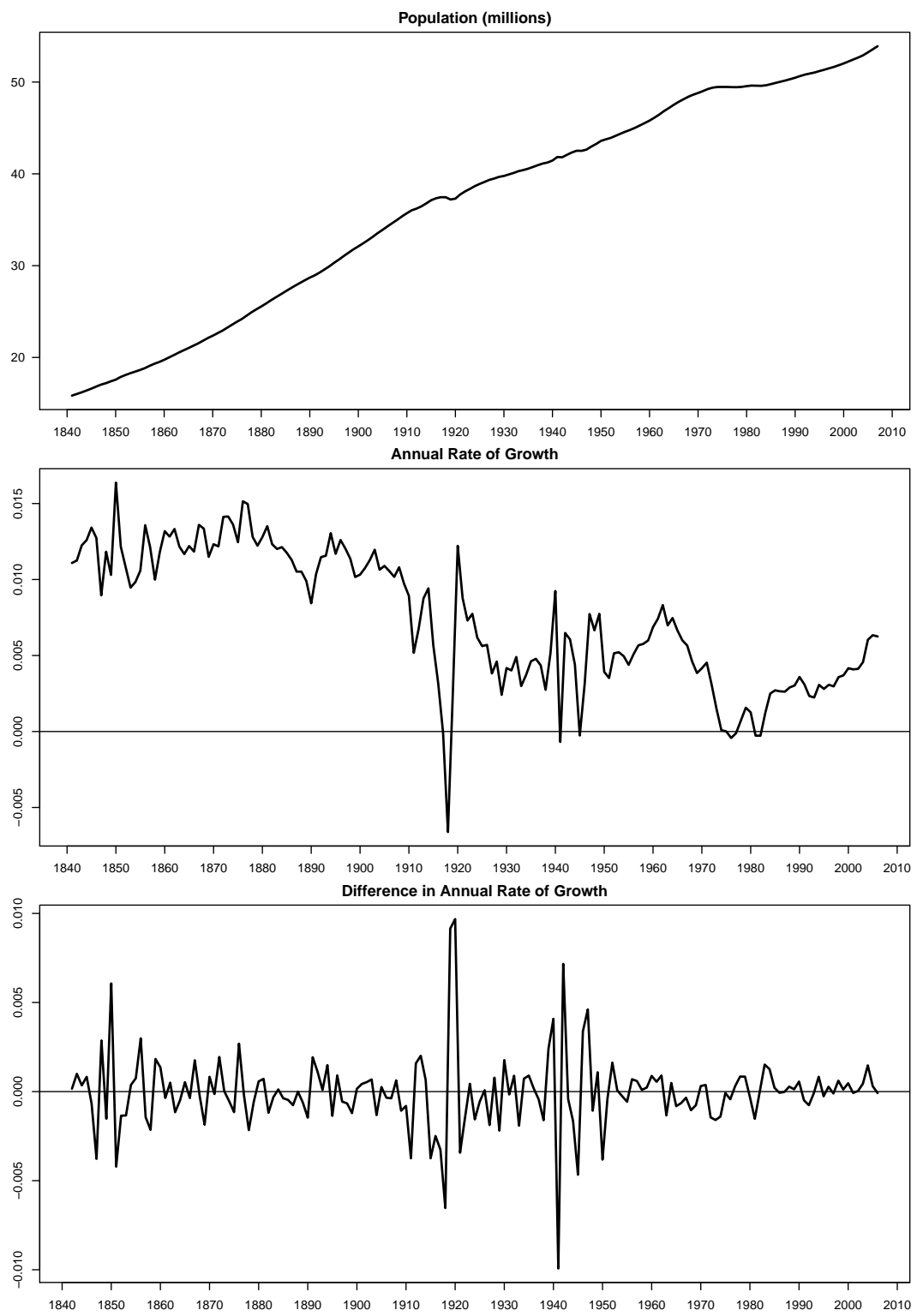


\section{Statistical background}

In this section, the models and notation used to forecast future annual growth rates in England and Wales are specified. The subsections introduce the autoregression models, stochastic volatility, random variance shifts, Bayesian inference and model uncertainty used in this paper.

Let $p_{t}$ be the population size at time $t$ for an uninterrupted series of observed time points. In population forecasting, we are interested in obtaining estimates of $p_{t}$ and the associated measures of uncertainty for one or more values of $t>T$, where $T$ is the last observed time point.

In order to model $p_{t}$, we first derive the time series of population growth rates $r_{t}$, where

$$
p_{t+1}=p_{t}\left(1+r_{t}\right) .
$$

Note, we focus on the geometric growth rate to be consistent with the measurement of the population data, which have been measured in annual increments. A time series plot of $r_{t}$ is presented in the second panel of Figure 1. It is clear that neither the mean nor the variance of this series are stationary. Therefore, as advocated by Chatfield (2003), we consider the changes in $r_{t}$ :

$$
y_{t}=r_{t}-r_{t-1} .
$$

A time series plot of $y_{t}$ for our data is presented in the third panel of Figure 1. It appears that an assumption of a stationary mean for $y_{t}$ is appropriate. The assumption of a stationary variance, however, is not justifiable. Hence, we need models to account for non-constant conditional variances, as presented in Section 3.2 and 3.3 below.

\subsection{Autoregression model}

There are several examples in the literature of autoregression (AR) models being used to forecast populations; see for example, Saboia (1974), Ahlburg (1987), Pflaumer (1992), Alho and Spencer (2005) and Tayman, Smith, and Lin (2007). An AR model of order $p$, denoted $\operatorname{AR}(p)$, is defined as

$$
y_{t}=\sum_{j=1}^{p} \phi_{j} y_{t-j}+z_{t},
$$

where $\phi_{j}$ are the autoregressive coefficients representing the correlations between observations $y_{t}$ and $y_{t-j}$, whilst $j$ represents the time lag, and $z_{t}$ are assumed to be independent 
observations from a probability distribution with zero mean and constant variance, $\sigma^{2}$. For a fully-specified probability model, a normal distribution for $z_{t}$ is typically assumed.

\subsection{Stochastic volatility model}

Stochastic volatility (SV) models have been widely used for modelling financial data, where the assumption of constant variance for $z_{t}$ is usually untenable. Models that account for non-constant variance have been sparsely used in the demographic context (Keilman and Pham 2004; Bijak 2010). This is surprising as historical time series of demographic data often exhibit volatility due to events such as epidemics, wars or baby booms. This is certainly true for the data presented in Figure 1.

SV models are time series models, similar to the AR models defined in (3), but where the variance of $z_{t}$ is allowed to be time-dependent. This is achieved by replacing $\sigma^{2}$ with $\sigma_{t}^{2}$, and specifying a time series model for $\sigma_{t}^{2}$. In this paper, we assume an $\operatorname{AR}(1)$ model for $\log \sigma_{t}^{2}$. Here, let

$$
\sigma_{t}^{2}=e^{h_{t}}
$$

and

$$
h_{t}=\psi_{0}+\psi_{1}\left(h_{t-1}-\psi_{0}\right)+\eta,
$$

where $h_{t}$ represents the volatility at time $t$ conditional on its own past, $\psi_{0}$ denotes the mean level of $h_{t}$ over the entire time period whilst $\psi_{1}$ is the autoregressive coefficient representing the correlations between $h_{t}$ and $h_{t-1}$. Finally, $\eta$ are the error terms of $h_{t}$ which are assumed to be independent observations from a normal distribution with zero mean and constant variance $\tau^{2}$. Other approaches to deal with non-constant variances include, for example, autoregressive conditional heteroscedastic models (ARCH) and generalised ARCH models (GARCH); see for example Chatfield (2003). All of these approaches assume that the variance changes at each time step. An alternative approach, which we consider next, is to adopt a model where the variance changes at less frequent intervals, with intervening periods of constant variance.

\subsection{Random variance shift model}

Random variance shift (RV) models, based on the models introduced by McCulloch and Tsay (1993), allow the standard deviations of $z_{t}$ to shift at certain time points by a ran- 
domly distributed factor, which we denote by $e^{\beta_{t}}$, so

$$
\sigma_{t}= \begin{cases}\sigma_{t-1} & \text { if } \delta_{t}=0 \\ e^{\beta_{t}} \sigma_{t-1} & \text { if } \delta_{t}=1\end{cases}
$$

where $\delta_{t}$ is a binary variable taking the value 1 if a variance shift occurs at time $t$ and 0 otherwise. We model the sequence of $\delta_{t}$ as independent Bernoulli random variables with $P\left(\delta_{t}=1\right)=\epsilon$, where $\epsilon$ is the probability of the shift. The magnitude of a variance shift, when it occurs, depends on the variable $\beta_{t}$ which is assumed to follow a normal distribution with mean 0 , and standard deviation $\lambda$ representing the magnitude (on the $\log$ scale) of average variance shifts. The key feature of this model is that it adapts to periods of high or low volatility; thus forecasts of uncertainty during a period of low volatility will be predominately based on a lower variance than the overall level.

\subsection{Bayesian inference}

In Bayesian inference, uncertainty about the (multivariate) parameter $\theta$ of a statistical model is described by its posterior probability distribution given observed data $y_{\{T\}}=$ $\left\{y_{1}, \ldots, y_{T}\right\}$. The probability density function of $y_{t}$ is obtained by using Bayes Theorem:

$$
f\left(\theta \mid y_{\{T\}}\right)=\frac{f\left(y_{\{T\}} \mid \theta\right) f(\theta)}{f\left(y_{\{T\}}\right)},
$$

where $f\left(y_{\{T\}} \mid \theta\right)$ is the likelihood function and is defined by the model, $f(\theta)$ is the prior distribution for $\theta$ and $f\left(y_{\{T\}}\right)$ is a normalising constant. The prior distribution $f(\theta)$ specifies the uncertainty about $\theta$ prior to observing any data.

Forecasting or prediction is particularly natural in a Bayesian framework. Uncertainty about the next $K$ future values of $y_{t}$ (for $t=T+1, \ldots, T+K$ ) is described by the joint predictive probability distribution

$$
f\left(y_{T+1}, \ldots, y_{T+K} \mid y_{\{T\}}\right)=\int f\left(\theta \mid y_{\{T\}}\right) \prod_{k=1}^{K} f\left(y_{T+k} \mid y_{\{T+k-1\}}, \theta\right) \mathrm{d} \theta .
$$

Note that the product term represents the joint predictive distribution in the case that parameter $\theta$ is known. The Bayesian predictive distribution simply averages (integrates) this with respect to the posterior probability distribution for $\theta$. Hence, uncertainty about $\theta$ in light of the observed data is fully incorporated.

In Bayesian analyses, forecasts and associated measures of uncertainty are obtained by calculating marginal probability distributions for quantities of interest by integrating 
the posterior distribution in (7) or the predictive distribution in (8). Performing these integrations analytically is often not possible for complex models, such as those described above. Historically, this has prevented demographers and others from taking advantages of Bayesian methods for statistical inference. More recently, this has become less of an obstacle as developments in Bayesian computation have improved. In particular, Markov chain Monte Carlo (MCMC) methods for generating samples from distributions, such as (7) or (8), have made it possible to apply Bayesian techniques to a wide variety of applications. Once a sample has been obtained from a joint distribution, then a sample from a distribution of any component or function of components is readily available; see Gelman et al. (2003) for details. To generate the samples from the posterior and predictive distributions for our study, we used the MCMC sampling approach implemented in the OpenBUGS software (Lunn et al. 2009). For each model, the performance of the MCMC sampler was assessed by standard visual inspection of time series traces of key parameters. After discarding any iterations required as burn-in, posterior summaries and projections were based on an MCMC sample of size of 10,000. Details on the code to formulate the Bayesian time series models for OpenBUGS used in this paper are provided in the next section.

\subsection{Model uncertainty}

It is unrealistic for the analyst to be sure that any particular statistical model is the correct one upon which to base their forecasts. Hence, the statistical methodology adopted should be one which allows for model uncertainty. Furthermore, we consider it essential that the measures of uncertainty associated with any forecast should incorporate both the uncertainty concerning the model and the uncertainty concerning the parameters of each model. Measurement error uncertainty in $r_{t}$ is practically insignificant relative to the size of the population. In this paper, model uncertainty is directly integrated with parameter uncertainty into a single predictive probability distribution. A comprehensive review of Bayesian model averaging is available in Hoeting et al. (1999), and for some demographic applications, see Bijak (2010) and Bijak and Wiśniowski (2010).

Formally, let $m=1, \ldots, M$ index the models under consideration and let $\theta_{m}$ represent the parameter associated with model $m$. Note that different models may have parameters of different dimensionality. For example, the $\operatorname{AR}(2)$ model has a three-dimensional parameter $\left(\phi_{1}, \phi_{2}, \sigma^{2}\right)$. The likelihood function for model $m$ is $f\left(y_{\{T\}} \mid \theta_{m}, m\right)$, the prior distribution for $\theta_{m}$ is $f\left(\theta_{m} \mid m\right)$ and the posterior distribution is

$$
f\left(\theta_{m} \mid y_{\{T\}}, m\right)=\frac{f\left(y_{\{T\}} \mid \theta_{m}, m\right) f\left(\theta_{m} \mid m\right)}{f\left(y_{\{T\}} \mid m\right)},
$$


where $f\left(y_{\{T\}} \mid m\right)$ is a normalising constant, known as the marginal likelihood for model $m$, and is given by

$$
f\left(y_{\{T\}} \mid m\right)=\int f\left(\theta_{m} \mid m\right) f\left(y_{\{T\}} \mid \theta_{m}, m\right) \mathrm{d} \theta_{m} .
$$

Prior uncertainty about models is encapsulated by a discrete probability distribution, $f(m), m=1, \ldots, M$. As we have no prior reason to prefer any model over any other, we give every model the same prior probability, $1 / M$.

The posterior probability distribution for $m$ given observed data $y_{\{T\}}$ is obtained by using Bayes Theorem:

$$
f\left(m \mid y_{\{T\}}\right)=\frac{f\left(y_{\{T\}} \mid m\right) f(m)}{f\left(y_{\{T\}}\right)} .
$$

Hence, the posterior model probability for any model $m$ is proportional to the product of the prior model probability and the marginal likelihood. Therefore, an efficient method for computation of marginal likelihoods is essential for Bayesian inference under model uncertainty (see, for example, those described in O'Hagan and Forster 2004). In our implementation, we found that the bridge sampler (Meng and Wong 1996) was effective for this computation.

Finally, to obtain a predictive distribution for population forecasts in the presence of model uncertainty, (8) is extended to

$$
\begin{aligned}
f\left(y_{T+1}, \ldots, y_{T+K} \mid y_{\{T\}}\right)= & \sum_{m=1}^{M} f\left(m \mid y_{\{T\}}\right) f\left(y_{T+1}, \ldots, y_{T+K} \mid y_{\{T\}}, m\right) \\
= & \sum_{m=1}^{M} f\left(m \mid y_{\{T\}}\right) \int f\left(\theta_{m} \mid y_{\{T\}}, m\right) \\
& \prod_{k=1}^{K} f\left(y_{T+k} \mid y_{\{T+k-1\}}, \theta_{m}, m\right) \mathrm{d} \theta_{m}
\end{aligned}
$$

which is the average of predictive distributions for individual models weighted by their posterior probabilities, $f\left(m \mid y_{\{T\}}\right)$.

\section{Computation and forecasts}

In this section, we illustrate the parameter estimation from a range of individual AR, $\mathrm{SV}$ and RV models. The predictive probability distributions from a selection of these 
models are provided in order to gain a better understanding of the effect of increasing the complexity of the model on future population growth rates. These individual forecasts are compared in Section 4.4 with a single forecast that incorporates uncertainty in model choice.

\subsection{Individual autoregressive models}

An initial set of nine models was considered for the differenced population growth rate, $y_{t}$, introduced in (1) and presented in the bottom panel of Figure 1. These consist of an independent normal (IN) model and eight autoregression models, increasing in order from $\mathrm{AR}(1)$ to $\mathrm{AR}(8)$. This range of models was selected in order to represent all possible autoregressive processes that might adequately describe the differences in the overall growth rate series. As we have no previous knowledge about the nature of the parameters in each model we assigned weakly informative prior distributions: $\phi_{j} \sim N(0,1), j=1, \ldots, p$ and $\sigma^{-2} \sim \operatorname{Gamma}\left(10^{-6}, 10^{-6}\right)$, where $N\left(\mu, \sigma^{2}\right)$ denotes a normal (Gaussian) distribution with mean $\mu$ and variance $\sigma^{2}$, and $\operatorname{Gamma}(a, b)$ denotes a gamma distribution with shape parameter $a$ and scale parameter $b$. As we also have no prior belief that the data should conform to a stationary regime, our prior distribution on the autoregression $\left(\phi_{j}\right)$ parameters does not constrain the model to be stationary.

The computation of AR models were undertaken in OpenBUGS, where BUGS models were produced using the ar.bugs function in tsbugs $\mathrm{R}$ package (Abel 2013). For example, a BUGS model for an AR(2) process on the difference of the population growth rate was created in $\mathrm{R}$ using the following code:

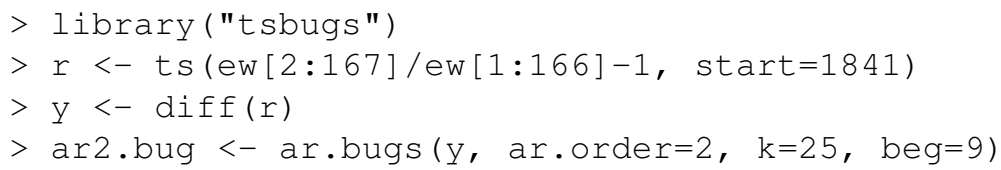

In the first three lines of above code, the differenced series of the population growth rate is derived as in (1) and (2), where ew is a time series object of the England and Wales data, contained in the tsbugs package. The fourth line uses the ar.bugs function to create the BUGS model. Arguments are set for the model order, the number of future data points to be forecast $(\mathrm{k})$ and the starting points for which the mean part of the likelihood should be calculated over (beg). This is set to 9 for all time series models run in our analysis, enabling the same set of data to be considered regardless of the number of autoregressive terms. The BUGS model in the ar2 object can be viewed using the 


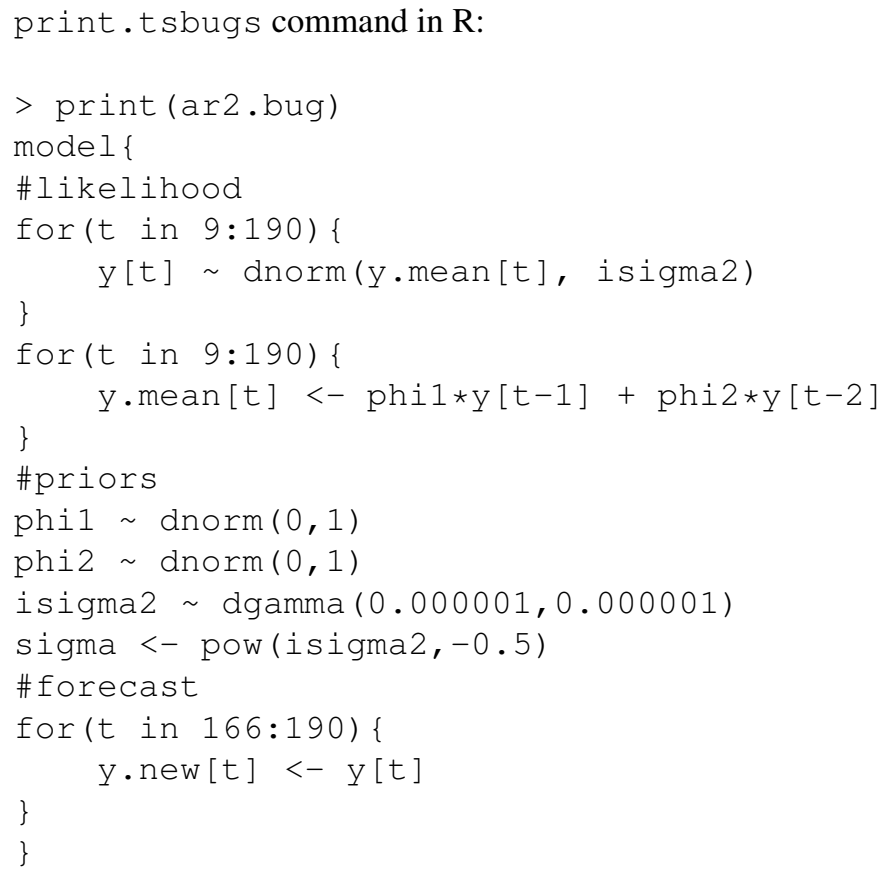

In the first part of the BUGS code, the likelihood for the AR(2) model is given. The random variable $y[t]$, defined in the first for loop is specified to come from normal distribution with mean $y \cdot$ mean [t] (defined in the second for loop) and precision isigma2. The likelihood is based on data points y [9] to y [190], where the last 25 observations are treated as missing by BUGS and simulated values given the parameters estimates are generated. In the second part of the BUGS code, the prior distributions are given, which by default are created with the distributions stated at the beginning of this subsection. In the third part of the BUGS code, forecasted values of $y_{t}$ that are estimated in the likelihood part of the model, are duplicated and relabelled $y$. new to enable an easier handling of the BUGS output.

The ar 2 . bug object in $\mathrm{R}$ has two additional elements, aside from the \$bugs element, that are not printed. First, the info element provides additional information on the data and BUGS model for use in other functions. The second is a cleaned version of the data formatted for use in R2OpenBUGS (discussed below). This is stored in the \$data element which can be displayed in $\mathrm{R}$ :

$>$ ar2.bug $\$$ data 
Abel et al.: Integrating uncertainty in time series population forecasts

$\$ y$

[1] $\quad \begin{array}{llllll}1.598748 e-04 & 9.949415 e-04 & 3.466781 e-04 & 8.274713 e-04 & -6.824515 e-04\end{array}$

[6] $-3.774363 e-03 \quad 2.865602 e-03-1.522894 \mathrm{e}-03 \quad 6.070610 e-03-4.213722 \mathrm{e}-03$

...

[161] $3.303926 \mathrm{e}-05 \quad 4.433711 \mathrm{e}-04 \quad 1.470939 \mathrm{e}-03 \quad 3.009411 \mathrm{e}-04 \quad-8.359634 \mathrm{e}-05$

[166]

NA

NA

NA

NA

NA

[181]

[186]

NA

NA

NA

NA

NA

NA

NA

NA

NA

Note, the output above is edited to reduce space. The $\$$ data is a list named $y$, to correspond to the 165 observed data points appended with 25 further missing data points to be forecast by the BUGS models.

Creating the BUGS model in R has two prominent advantages. Firstly, users can easily run the estimation in OpenBUGS using the R2OpenBUGS package (Sturtz, Ligges, and Gelman 2005). Secondly, numerous output analysis and diagnostics for the MCMC simulations are available in the coda package (Plummer et al. 2006). The BUGS model produced by the code above can be passed to OpenBUGS in R:

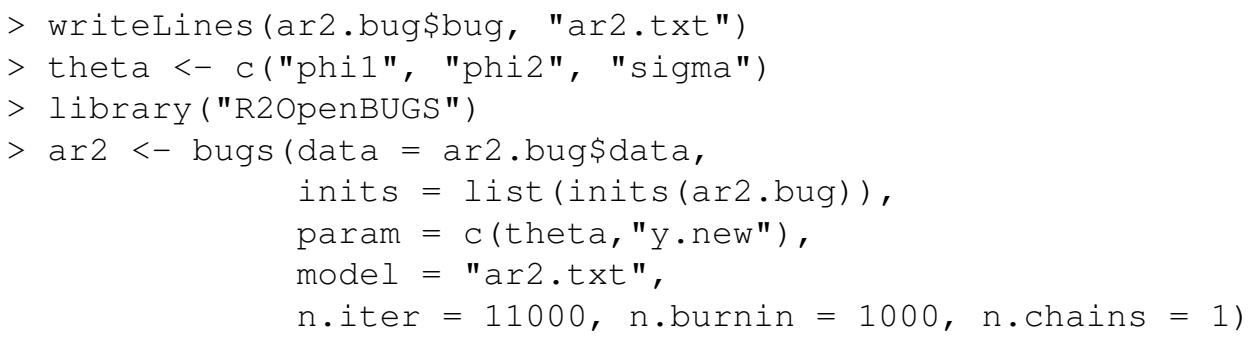

In the first line of the code above, the BUGS code, stored in the \$bug element of the ar2. bug object, is written to text file in the local directory. In the second line the object theta is created to correspond the $\operatorname{AR}(2)$ parameter set for later use. In the rest of the code, the bugs command in the R2OpenBUGS package is used to fit the BUGS model, given the specified arguments for the data, initial values, parameters to monitor in the MCMC simulations, the name of the model text file and MCMC settings for run lengths and burn in periods. Functions in the tsbugs package can help provide the values of the first two arguments. Initial values can be automatically generated for the parameters in the tsbugs model using the inits function. The parameters names are concatenated with the $\mathrm{y}$. new variable to specify that simulations of the both the parameters and forecasted values should be returned. The results of the MCMC simulations are stored in the ar2 object. The convergence of the MCMC can be analysed using various convergence 
diagnostics in the coda package. For example, the trace plots in Figure 2 of the model parameters can be obtained after converting the posterior simulations to an mcmc type object:

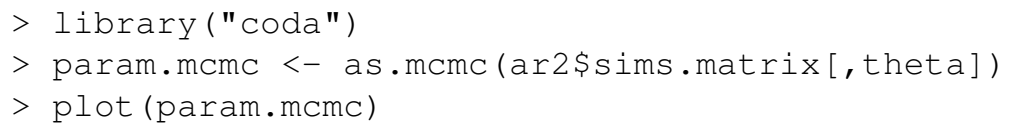

In the appendix of this paper, we illustrate how the density function of prior distributions can be plotted on top of the posterior densities from the plot. mcmc function.

\section{Figure 2: $\quad$ Trace plots and posterior distributions of AR(2) model parameters}
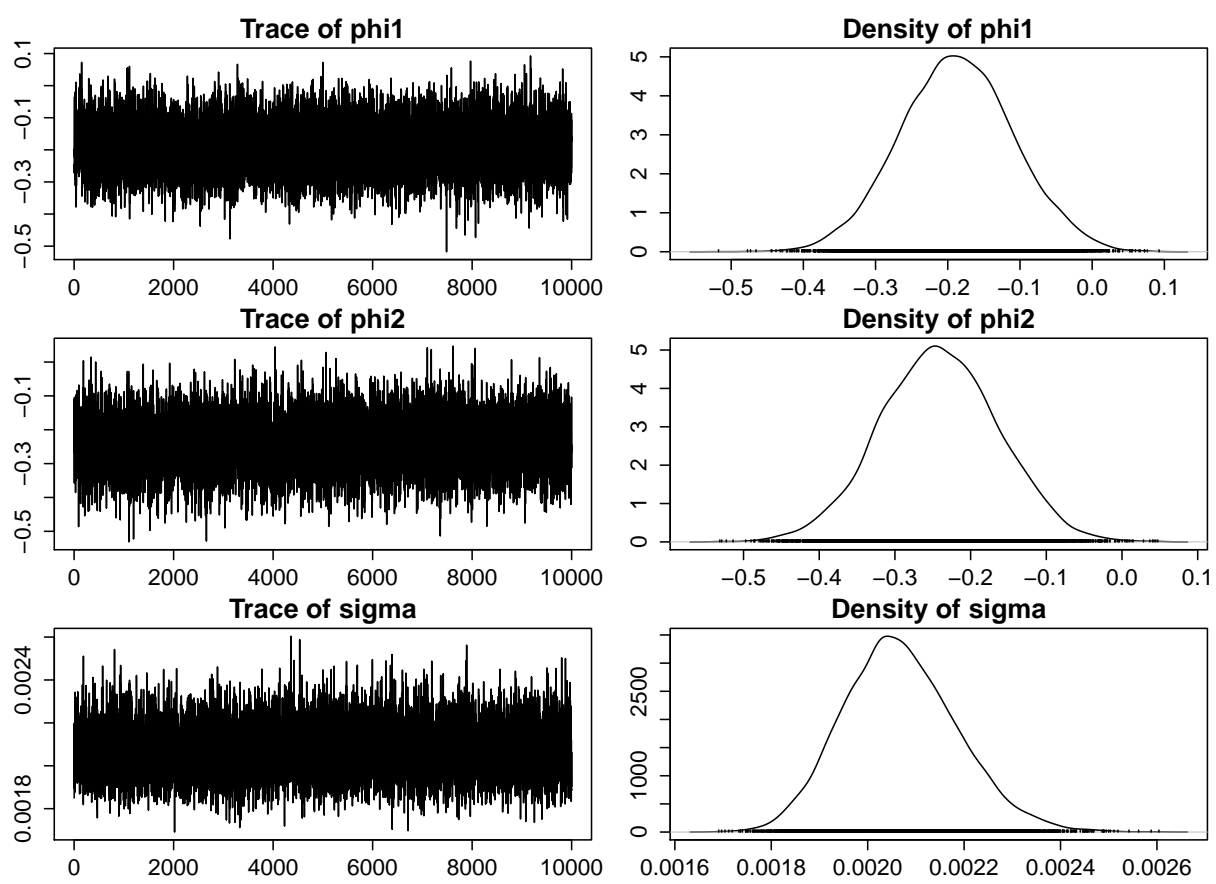

The marginal posterior distributions, such as those in Figure 2, completely describe the uncertainty about each model parameter given the observed data. In a Bayesian anal- 
ysis, these are typically summarised by using posterior means (as parameter estimates) and posterior standard deviations (as measures of uncertainty), which we calculated using the summary. mcmc command in the coda package. For all AR models, the posterior means of $\sigma$ are around 0.002 with much lower standard deviations than in their prior distributions. In all models, the posterior means of $\phi_{j}$ at lower values of $j$ were below zero, indicating negative autocorrelation for these lags. Estimates of $\phi_{j}$, for $j>5$, tend to be close to zero, signifying that the association between $y_{t}$ and $y_{t-j}$ becomes weak at larger values of $j$.

Posterior predictive plots of the forecasted $r_{t}$ from the IN, AR(4) and AR(8) models are illustrated in the top row of Figure 3, where the darkest shades correspond to higher probability masses of the posterior distributions. Contour lines are also plotted at each decile. Forecasts from the simple independent normal (IN) model resulted in a higher level of uncertainty in future values than did any of the AR models. As autoregressive parameters were added to the independent normal model, the posterior predictive distribution became comparatively narrower.

Plots of the posterior predictive distributions can be replicated using the fanplot package (Abel 2012) in R and the MCMC results stored in a bugs object:

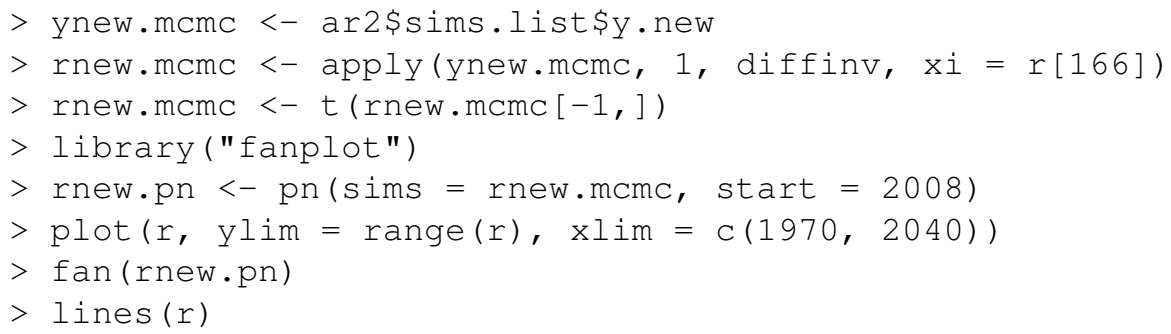

The first three lines of this code use the $y$. new [ $t$ ] variables in the BUGS model and the last observed value of $r_{t}$ to derive the simulated values of posterior predictive distribution via the diffinv function. The rest of the code calculates the percentiles of the posterior predictive distributions for $r_{t}$ with the pn function and then plots the percentile object using the fan function in the fanplot library. 
Figure 3: $\quad$ Posterior predictive plots of population growth rates from selected constant variance, stochastic volatility and random variance shift models
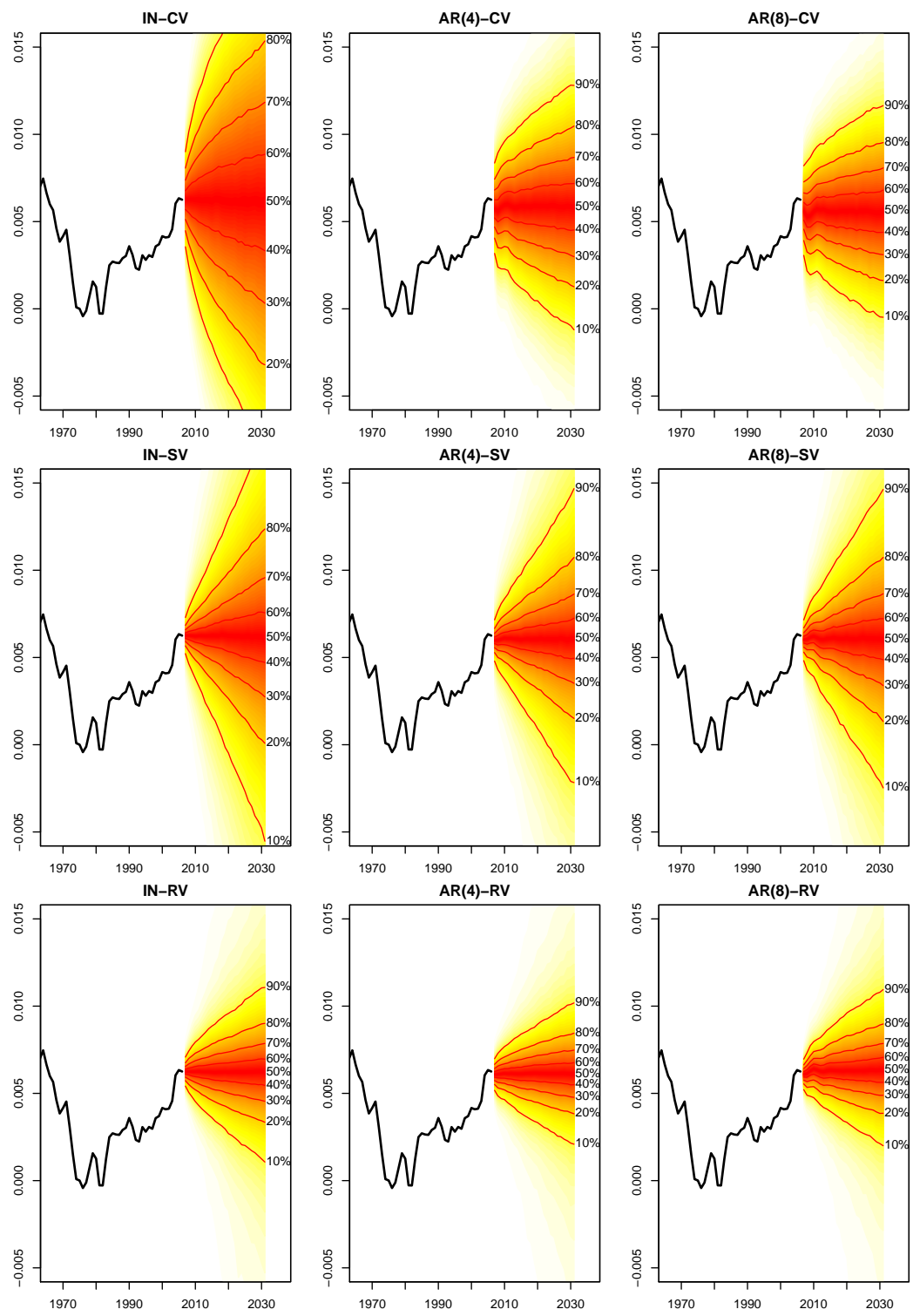


\subsection{Individual stochastic volatility models}

Nine SV models were considered for the differenced population growth rate. The SV extension replaces the $\sigma^{2}$ term in the AR models with time dependent variances $\sigma_{t}^{2}$. As specified in (5), this results in three new parameters $\psi_{0}, \psi_{1}$ and $\tau$, as well as values of $h_{t}$ at each time point. Weakly informative prior distributions were also assigned to the new parameters: $e^{-\psi_{0}} \sim \operatorname{Gamma}\left(10^{-6}, 10^{-6}\right), \psi_{1} \sim U(-0.999,0.999)$ and $\tau^{-2} \sim$ Gamma $(0.01,0.01)$. The BUGS models were again produced using tsbugs package. For example, a BUGS model for an AR(2)-SV process was created in R:

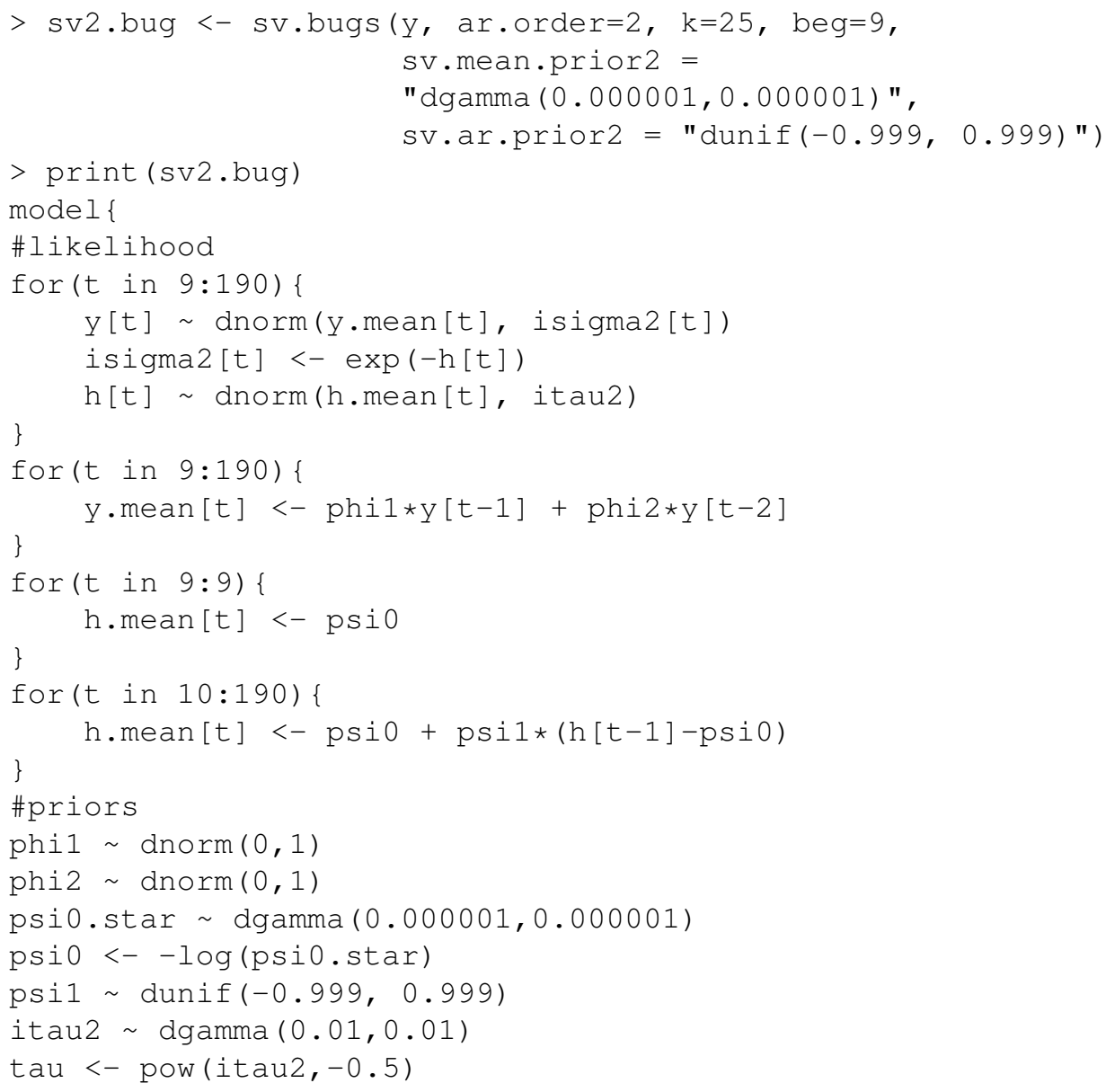




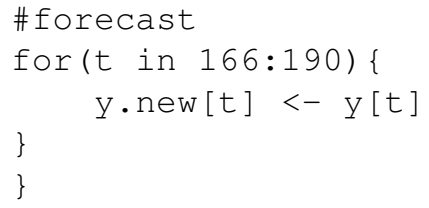

In the first loop of the BUGS model, the likelihood of the AR(2)-SV model is defined in a similar fashion as that of the constant variance equivalent shown previously. However, the precision term for $y_{t}$, isigma 2 [ $t$ ] has a index allowing for a variation over time. This is formed by a transformation of the volatility process $h[t]$. In the second loop the AR(2) mean process is set up. In the third and fourth loops the mean level of the volatility process is given an $\mathrm{AR}(1)$ process for all but the initial data point. The likelihood part of the BUGS code is followed by the prior distributions which are specified in the $\mathrm{SV}$. bugs command to take the distributions presented at the beginning of this subsection (the default values for these arguments are different). Finally, a new set of variables is created to record the forecasted values. Plots of the MCMC traces, posterior and prior distributions of the AR(2)-SV model are shown in the appendix.

As with the constant variance models, the fitting of the parameters for the SV models by OpenBUGS were run from R, allowing MCMC diagnostics checks and summary statistics of the posterior distributions to be easily obtained. The posterior means and standard deviations of the parameters in the nine SV models are presented in Table 1. Estimates of autoregressive parameters tend to be close to zero for most $\phi_{j}$ with the exception of $j=2$ and $j=3$. The posterior means of $\psi_{0}$, the average volatility level, are similar across all models. The corresponding values of the variance $\left(e^{\psi_{0}}\right)$ are very similar to those for the AR models with constant variance. Posterior means for $\psi_{1}$, representing the autocorrelation between a current level of volatility and that of a previous year, are all close to 0.92 . This indicates a strong positive autocorrelation in the volatility levels of $r_{t}$. Estimates of $\tau$, measuring the standard deviation of volatility, are also similar across all models. 
Table 1: $\quad$ Posterior means (standard deviations) of stochastic volatility model parameters from MCMC simulations

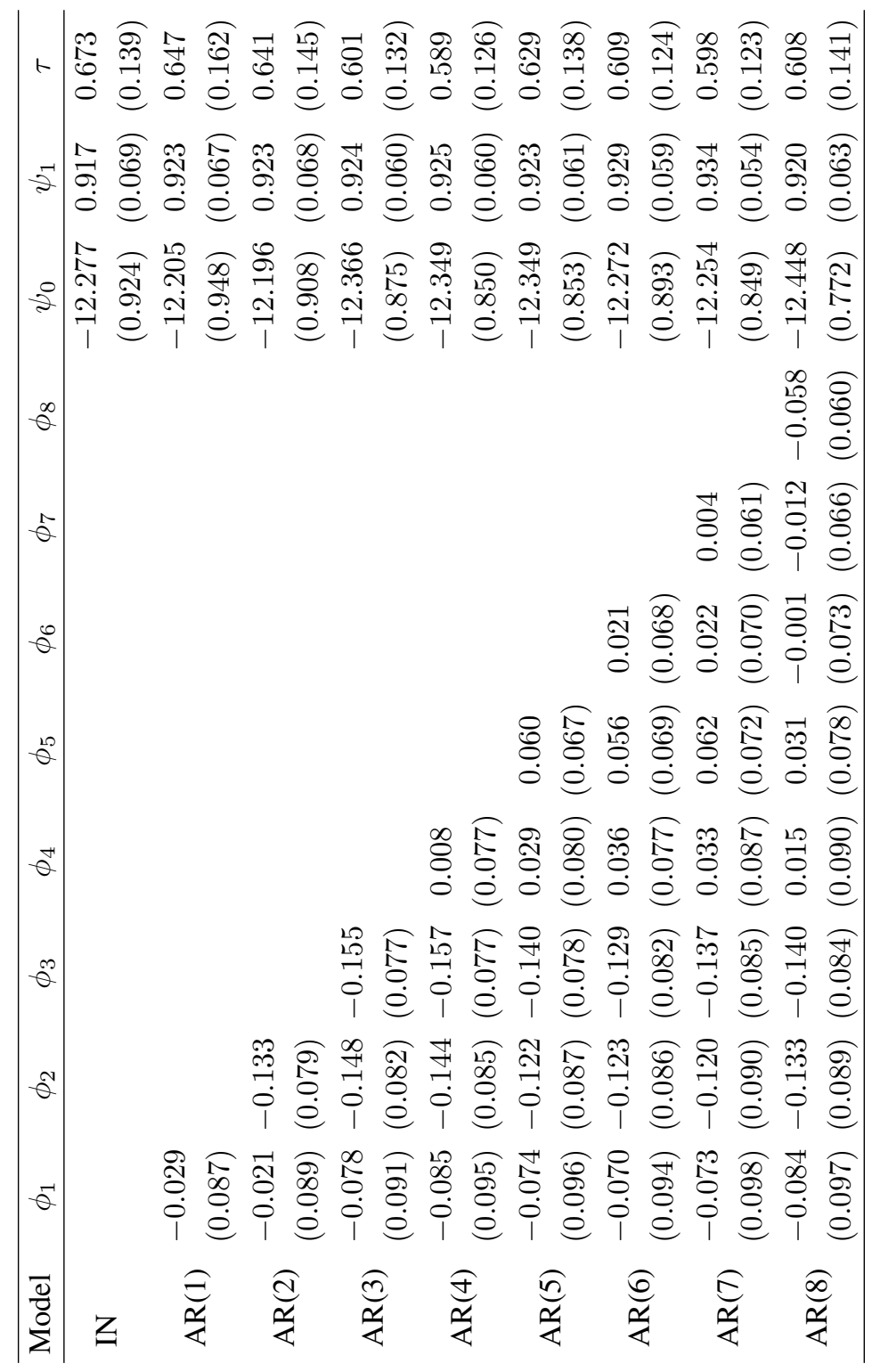


The posterior distributions for the $\sigma_{t}$ parameters, obtained by transforming the precision values (isigma2 [t] from OpenBUGS), are plotted in the upper panel of Figure 4 for the independent normal model with stochastic volatility (IN-SV). These were produced in a similar fashion as the forecast fans in Figure 3. Inspection of this plot reveals a number of features. First, the estimated standard deviations decrease throughout most of the observed period. Volatility is at its lowest level in 2001, prior to a increase in subsequent years leading up to the last observation, marked by the vertical line. Second, the estimated standard deviations are highest during the 1918 influenza pandemic and war periods. During these years, the 10th percentiles of the estimated standard deviation are higher than the 90th percentiles for 2007. Finally, the median and width of the predictive distributions gradually increases over time.

\section{Figure 4: $\quad$ Posterior and predictive distributions of standard deviation $\left(\sigma_{t}\right)$ from the IN-SV model (upper panel) and the IN-RV model (lower panel)}
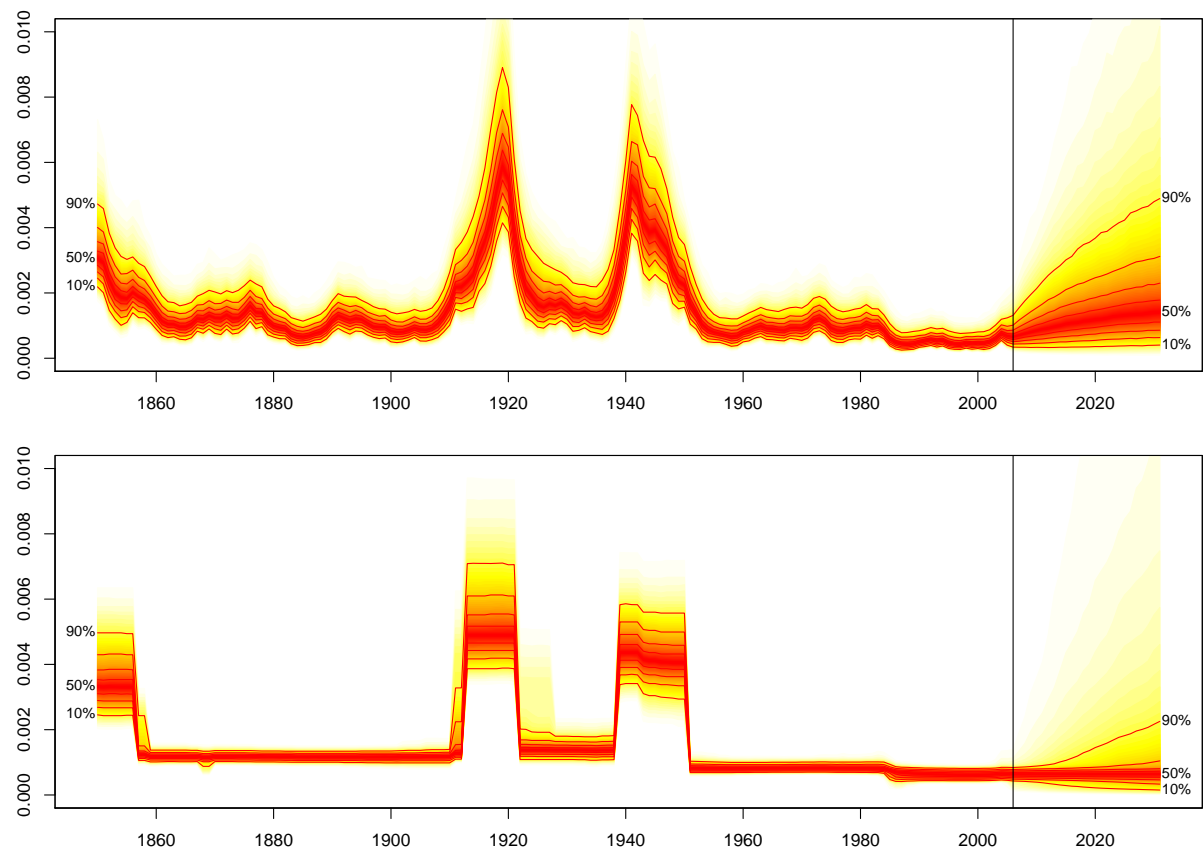
Posterior predictive plots of the forecasted $r_{t}$ from the IN-SV, AR(4)-SV and AR(8)$\mathrm{SV}$ models are presented in the middle row of Figure 3. Comparisons between models with SV terms reveal that uncertainty in forecasted $r_{t}$ is reduced through the addition of autoregressive parameters, as was the case with the AR models with constant variance. However, for $j>3$, the reductions in uncertainty were minimal as the values of $\phi_{j}$ remained close to zero. Comparison of the forecasted population growth rates between the selected individual models with constant variance and the SV models (between the top and middle row in Figure 3) demonstrates a different shape in the forecast fans, caused by a combination of lower $\phi$ values and additional terms for a non-constant variance in the SV models. The inter-decile ranges of the predictive distributions in the SV models increase at a steady rate. The corresponding contour lines in the constant variance models, on the other hand, tend to spread quickly (depending on the order of the AR model) and then continue to widen at a steady but slower rate. For the IN model, the inclusion of the SV terms reduces the width of the predictive distribution, as illustrated in 2032 where the difference between the 90th and 10th percentile is 0.028 for the IN-CV model compared to 0.023 for the IN-SV model. This is because the SV model acknowledges the recently observed low volatility in its forecast.

\subsection{Individual random variance shift models}

As with the SV models, RV models allow for a time dependent variance of $y_{t}$. The RV model replaces the $\psi_{0}, \psi_{1}, \tau$ and $h_{t}$ terms in the SV models with time constant parameters $\epsilon$ and $\lambda$, and time varying parameters $\beta_{t}$ and $\delta_{t}$; see (6). For the additional parameters in the RV model, we assigned the following prior distributions: $\beta_{t} \sim N\left(0, \lambda^{2}\right), \delta_{t} \sim$ $\operatorname{Bernoulli}(\epsilon)$ and $\lambda^{-2} \sim \operatorname{Gamma}(0.01,0.01)$. The prior distribution for $\epsilon \sim \operatorname{Beta}(1,100)$, where $\operatorname{Beta}(\mathrm{a}, \mathrm{b})$ denotes a beta distribution with shape parameter $a$ and scale parameter $b$, which is set to produce a low probability of a random variance shift. This reflects our prior belief that variance shifts should be relatively rare events.

Scripts to fit the models in OpenBUGS were produced using the rv. bugs command in the tsbugs package. For example, the AR(2)-RV model was created in R:

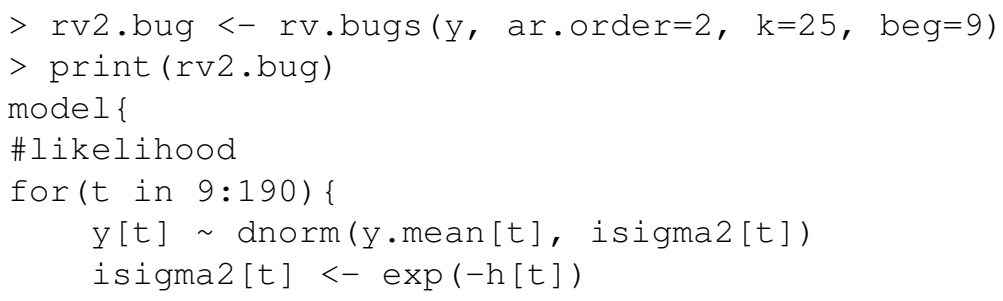




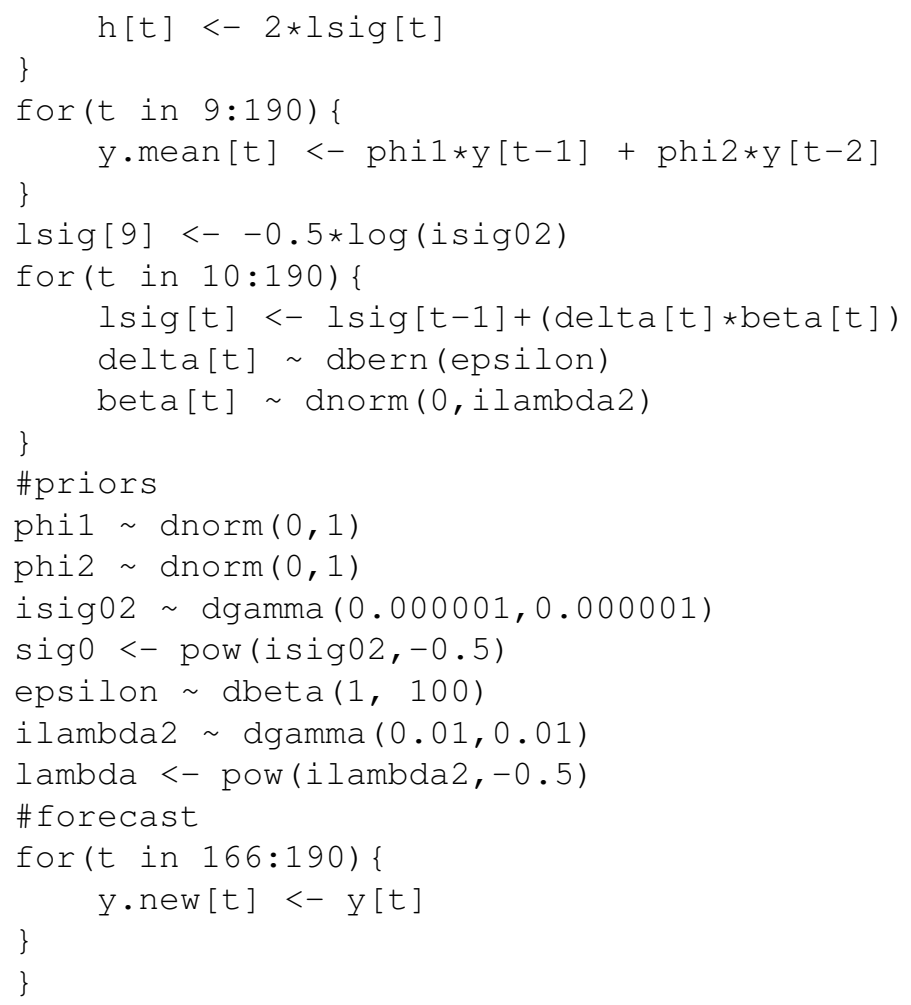

In the first loop of the BUGS code above, the likelihood of the AR(2)-RV model is defined similarly to the SV equivalent. However, the volatility, $h[t]$, which monitored for comparative purposes, is derived from transforming the logarithm of the standard deviation, lsig [t]. This variable is set up in the last loop of the likelihood and is equivalent the logarithmic transformation of (6). Preceding the final in the likelihood part of the BUGS code is a transformation to the logarithmic scale of the standard deviation for the prior distribution defined by isig02. This prior is required in order to initiate subsequent variation levels and random shifts. As with previous BUGS code, the prior distributions are specified after the likelihood. The rv. bugs command takes the prior distributions stated in the top of this subsection as default values. Finally, a new set of variables are created to record the forecasted values. Plots of the MCMC traces, posterior and prior distributions of the AR(2)-RV model are shown in the appendix.

The posterior means and standard deviations of the parameters in the nine RV models 
are presented in Table 2. Again, the estimates of the autoregressive parameters are close to zero for most $\phi_{j}$ with the exception of $j=2$ and $j=3$. The posterior means of $\epsilon$, i.e., the probability of a variance shift, are similar across all models with around a $4 \%$ chance of a variance shift in any given year during the observed period. The $\lambda$ parameter, representing the magnitude (on the log scale) of average variance shifts, also remains relatively constant across all models.

The posterior distributions for the $\sigma_{t}$ parameters are plotted in the lower panel of Figure 4 for the independent normal model with random variance shifts (IN-RV). In comparison to the SV plot in the upper panel, the standard deviations shift according to the $\delta_{t}$ exhibit considerably narrower posterior distributions during periods of low volatility. In the first period up to 1858 , the standard deviation is at a relatively high level, before a downward shift, followed by a period of stability at a lower level. The standard deviations increase dramatically during the times of the two wars and the 1918 influenza pandemic. The posterior distributions of the standard deviations immediately after the Second World War are slightly lower than during the conflict period. In the early 1950s the standard deviations narrow considerably and with a lower median level. The upper deciles of the predicted posterior standard deviations from the RV model, in the future time period, are much lower than those of the SV model.

Posterior predictive plots of the forecasted $r_{t}$ from the IN-RV, AR(4)-RV and AR(8)$\mathrm{RV}$ models are presented in the bottom row of Figure 3. In comparison to the corresponding SV models, the forecast uncertainty is much lower. For the IN model, the inclusion of the $\mathrm{RV}$ terms reduces the width of the predictive distribution even further (in comparison to the CV and SV models) with the difference between the 90th and 10th percentiles being 0.010 for the IN model. Similar to the CV and SV models, the uncertainty decreases from independent normal to $\mathrm{AR}(4)$ and then remains fairly stable. 
Table 2: $\quad$ Posterior means (standard deviations) of random variance shift model parameters from MCMC simulations

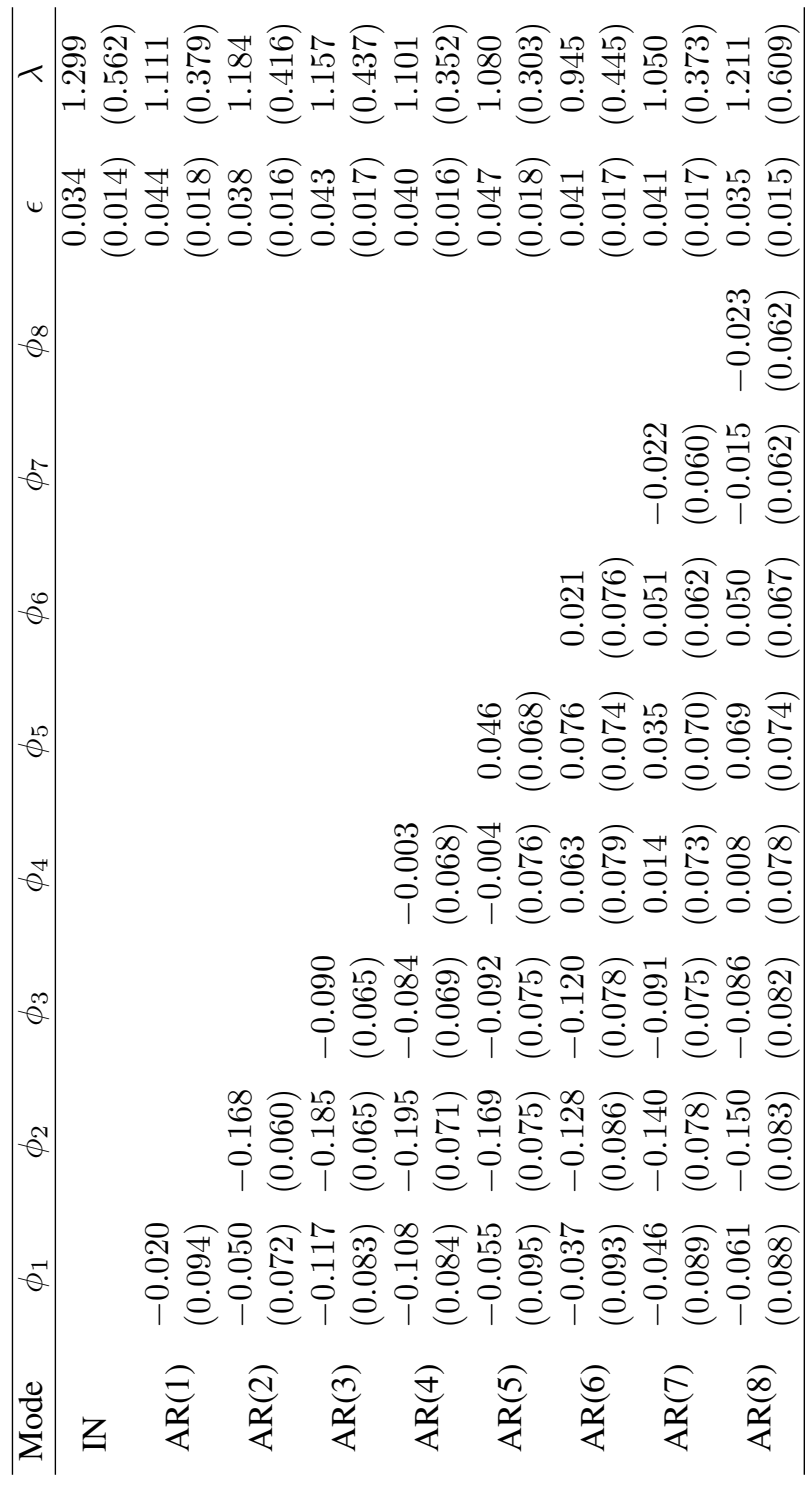




\subsection{Model-averaged forecasts}

We estimated posterior probabilities, $f\left(m \mid y_{\{T\}}\right)$, for all models, using the approach described in Section 3.5. These are displayed in the top panel of Table 3. Note, the posterior probabilities for the models with AR component of order 5 or higher were very close to zero and hence are aggregated. A replicable demonstration of the calculations, performed in R using the tsbridge package (Abel, G.J. and Wong, J.T.S. 2013), is provided in the online supplementary materials.

Over all 27 models, the posterior model probabilities give strong support both the IN-SV model and the AR(1)-SV model (posterior probabilities of 0.828 and 0.126 respectively). Other SV models have some small amount of support. All models with a constant variance and random variance shift terms appear very unlikely with posterior model probabilities close to zero.

The predictive probability distributions of $r_{t}$, averaged over all models, are presented in the top left hand panel of Figure 5. Because a sample from the posterior of probability distribution of each individual model is generated in the analysis, calculation of the averaged predictive probability distribution over all models is straightforward. Not surprisingly, this plot strongly resembles the IN-SV model forecast in Figure 3, for which large posterior model probabilities were obtained. However, one should keep in mind that it also includes a small amount of information from other SV models. On the top right hand panel of Figure 5, we present the resulting population forecasts from the predictive probability distributions of $r_{t}$. Our results provide a median predictive population of 62.9 million in 2032 with the 10th percentile at 55.2 million and the 90th percentile at 71.6 million.

A considerable contribution in the width of the $80 \%$ prediction intervals in the top panel of Figure 5 are generated by large tails in the forecast distributions, represented by the lighter, yellow colours. For example, the $60 \%$ prediction interval for the population in 2032 ( 9.0 million) is almost half the size of the $80 \%$ prediction interval (16.4 million). We found that smaller tails of forecasted distributions are produced if we dropped all stochastic volatility models from the anlaysis. These are shown in the bottom panel of Figure 5, where the medians are very close to those produced under all models. Forecasts are based predominately on a combination of the AR(2)-RV and IN-RV models with posterior probabilities of 0.558 and 0.440 respectively.

The decision to only consider RV models to account for non-constant variance might be of interest to potential user for a couple of reasons. First, the wider forecast distributions from the inclusion of the SV models originate in the different specification of the time-varying variance term, $\sigma_{t}$. In the SV models the size of the tails of the forecasted distribution are influenced by the $\tau$ parameter, for the variance of the volatility. Its estimate, and hence the forecasts are based on all past observations including periods of great 
changes in the volatility during and between the wars. In contrast, in the RV models the future uncertainty in the forecasted distribution is predominately dictated by the estimate of $\sigma_{T}$ in the last observed period of the differenced series. As the estimated level in the last period is at a historic low, see Figure 4, the smaller variance is carried through to the forecast. The RV model does allow for future increases (or decreases) from this estimated small variance, via $\epsilon$ the probability of a shift. However, as shown in Table 2 these probabilities were relatively low. Second, as illustrated in the next section, for recent in-sample forecasts made in low volatility periods they appear to be better calibrated.

Figure 5: Joint predictive probability distribution of the model averaged growth rates (left) and population forecast in millions (right) over all models (top panel) and only constant variance and random variance shift (bottom panel
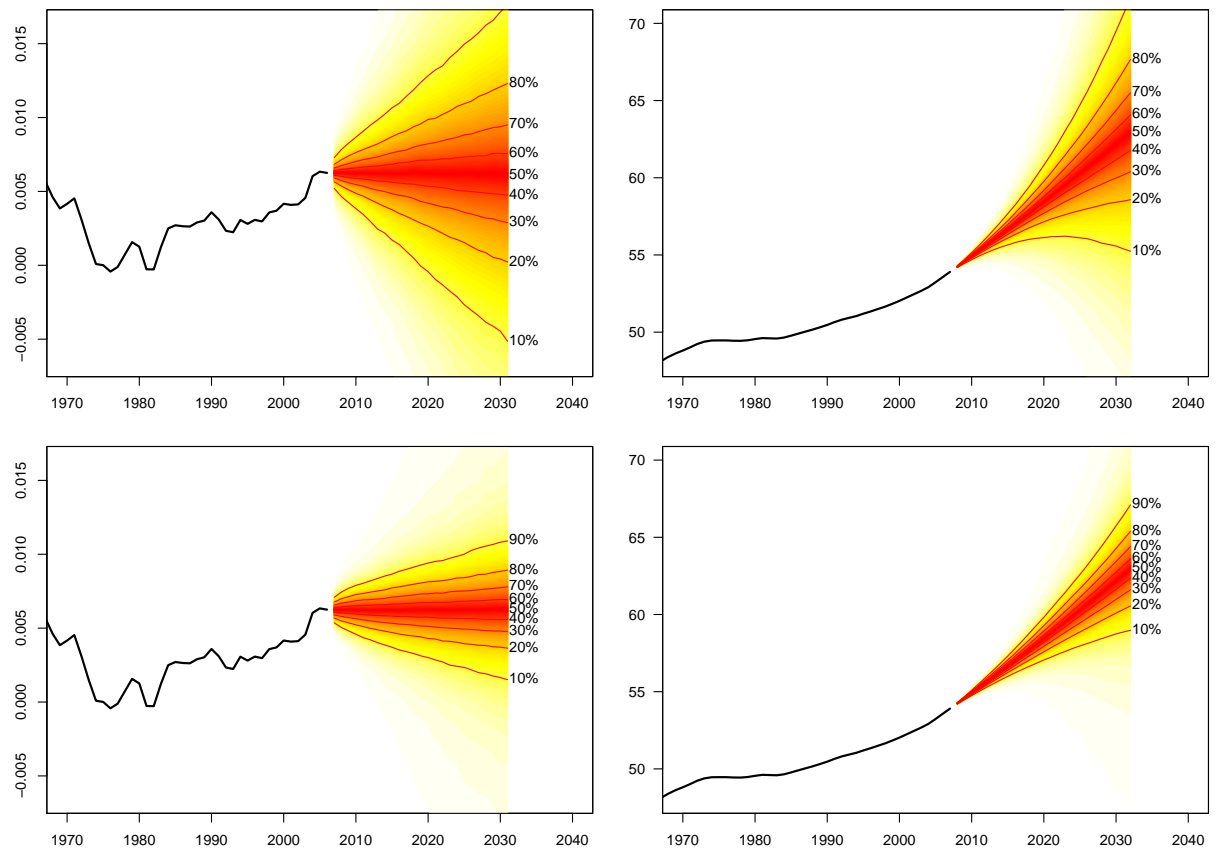
Table 3: $\quad$ Posterior model probabilities for both the full data series (top) and four reduced data series with different end points (bottom)

\begin{tabular}{|c|c|c|c|c|c|c|c|}
\hline \multicolumn{8}{|l|}{ Full Sample } \\
\hline Model & CV & SV & RV & & & & \\
\hline IN & 0.000 & 0.828 & 0.000 & & & & \\
\hline $\operatorname{AR}(1)$ & 0.000 & 0.126 & 0.000 & & & & \\
\hline $\mathrm{AR}(2)$ & 0.000 & 0.031 & 0.000 & & & & \\
\hline $\mathrm{AR}(3)$ & 0.000 & 0.015 & 0.000 & & & & \\
\hline $\operatorname{AR}(4)$ & 0.000 & 0.001 & 0.000 & & & & \\
\hline $\mathrm{AR}(5)-\mathrm{AR}(8)$ & 0.000 & 0.000 & 0.000 & & & & \\
\hline \multicolumn{8}{|c|}{ Truncated Samples } \\
\hline \multicolumn{4}{|c|}{ Last Data Point: 1900} & \multicolumn{4}{|c|}{ Last Data Point: 1925} \\
\hline Model & CV & SV & RV & Model & CV & SV & RV \\
\hline IN & 0.019 & 0.251 & 0.170 & IN & 0.000 & 0.661 & 0.073 \\
\hline $\operatorname{AR}(1)$ & 0.019 & 0.050 & 0.096 & $\operatorname{AR}(1)$ & 0.000 & 0.079 & 0.004 \\
\hline $\operatorname{AR}(2)$ & 0.003 & 0.023 & 0.084 & $\mathrm{AR}(2)$ & 0.000 & 0.107 & 0.001 \\
\hline $\operatorname{AR}(3)$ & 0.061 & 0.017 & 0.084 & $\operatorname{AR}(3)$ & 0.000 & 0.051 & 0.008 \\
\hline $\operatorname{AR}(4)$ & 0.030 & 0.007 & 0.069 & $\mathrm{AR}(4)$ & 0.000 & 0.012 & 0.002 \\
\hline $\operatorname{AR}(5)-A R(8)$ & 0.006 & 0.001 & 0.010 & $\operatorname{AR}(5)-A R(8)$ & 0.000 & 0.002 & 0.001 \\
\hline \multicolumn{4}{|c|}{ Last Data Point: 1950} & \multicolumn{4}{|c|}{ Last Data Point: 1975} \\
\hline Model & $\mathrm{CV}$ & SV & RV & Model & $\mathrm{CV}$ & SV & $\mathrm{RV}$ \\
\hline IN & 0.000 & 0.476 & 0.000 & IN & 0.000 & 0.744 & 0.000 \\
\hline $\operatorname{AR}(1)$ & 0.000 & 0.251 & 0.000 & $\operatorname{AR}(1)$ & 0.000 & 0.125 & 0.000 \\
\hline $\operatorname{AR}(2)$ & 0.000 & 0.119 & 0.000 & $\operatorname{AR}(2)$ & 0.000 & 0.060 & 0.000 \\
\hline $\operatorname{AR}(3)$ & 0.000 & 0.132 & 0.000 & $\operatorname{AR}(3)$ & 0.000 & 0.064 & 0.000 \\
\hline $\mathrm{AR}(4)$ & 0.000 & 0.019 & 0.000 & $\mathrm{AR}(4)$ & 0.000 & 0.007 & 0.000 \\
\hline $\mathrm{AR}(5)-\mathrm{AR}(8)$ & 0.000 & 0.002 & 0.000 & $\mathrm{AR}(5)-\mathrm{AR}(8)$ & 0.000 & 0.000 & 0.000 \\
\hline
\end{tabular}

The RV models tend to have lower posterior probabilities than the SV models, especially for longer series, as they require considerably more parameters in the specification of the $\sigma_{t}^{2}$. In the SV models, the $\sigma_{t}^{2}$ series are estimated using three time constant parameters $\left(\psi_{0}, \psi_{1}\right.$ and $\left.\tau\right)$ and another $T$ time varying $h_{t}$ parameters. The RV models has only two time-constant variables ( $\epsilon$ and $\lambda$ ) but twice the amount of time varying parameters in $\delta_{t}$ and $\beta_{t}$. The higher number of parameters tend to lead to a small posterior density for the RV models in comparison to the SV models, which in turn can lead to small poste- 
rior probabilities, when the observed data does not clearly support a variance-shift type structure. However, for the purposes of forecasting population, the RV models can potentially provide a more effective control for outlier events in the past data, such as war and epidemics.

\section{In-sample forecast validation}

In this section, we show how the posterior model probabilities and associated forecasts change when four different time periods are used to fit the models: 1841-1900, 18411925, 1841-1950 and 1841-1975. For each of the four time periods, we fitted the same 27 models described in Section 4 and then calculated the posterior model probabilities to produce model averaged forecasts. These in-sample forecasts were made for 25 years beyond the range of data used for fitting, to enable a comparison with actual observed rates, in order to validate the approach.

In the two bottom panels of Table 3, we present the posterior model probabilities for all 27 models fitted to each of the four time periods described above. As with the full sample in the top panel, the low posterior probabilities for models with AR orders of 5 and higher were aggregated for presentation purposes. For the first set of models, based on population data from 1841-1900, we find the IN-SV model has the largest model probability of 0.251 , followed by the IN-RV model, and then other SV and RV models. For this period, there was a small amount of support for the models with constant variance. For the second forecast, based on data from 1841-1925, the model probabilities indicate a large support for the IN-SV models with a probability of 0.661 . The third forecast, based on data from 1841-1950, resulted in the large model probabilities for IN-SV and AR(1)-SV models as was the case for the final forecast based on data from 1841-1975. In comparison to the model probabilities for the full sample forecast presented in the upper panel of Table 3, the forecasts with end points 1925, 1950 and 1975 are similar in that they all have considerable model probabilities for SV models, especially the IN-SV for the longer series. In all cases, there was very little support for the constant variance models.

To illustrate how the model averaged forecasts performed in relation to the observed data, we present the posterior predictive distributions for the four periods in the top panel of Figure 6.For the first forecast, several data observations appear in the tails of the forecast distributions, suggesting that the forecast uncertainty may be somewhat underestimated, due to the fact that, prior to 1900, there was no experience of a period of extremely high variability. The second and third forecasts, not surprisingly, exhibited considerably more uncertainty, as they were based on data which included recent periods of high variability. In the case of the third forecast, which took place just before a dramatic fall, the first future observation (the population growth rate in 1950) is still within the 5th 
percentile of the predictive distribution. For the last forecast, we see the uncertainty decreases somewhat, after the variance shifts to a period of relatively low volatility. Here, the observed data remain within the 30th and 70th percentiles.

The posterior predictive distributions for the four periods based only on constant variance and random variance shift models are shown in the bottom panel of Figure 6. The forecasted distributions are wider than those based predominately on the SV models for data cut at 1925 and 1950, due to higher variances levels estimated in the RV models during these base years. In the case of the series ending in 1925, there are too few observations beyond the war and pandemic to shift variance levels downwards, as suggested by models fitted to the entire data series (as in Figure 4). Conversely, as with the 2007 forecast in the previous section, they provide narrower forecasted distributions in low volatility periods. In the case of the forecast from the series ending in 1975 the forecast distribution appears to be better calibrated to future observed values that the forecasts based predominately on the SV models.

Figure 6: $\quad$ In-sample forecasts with data cut at 1900, 1925, 1950 and 1975 based on all models (top panel) and only constant variance and random variance shift (bottom panel)
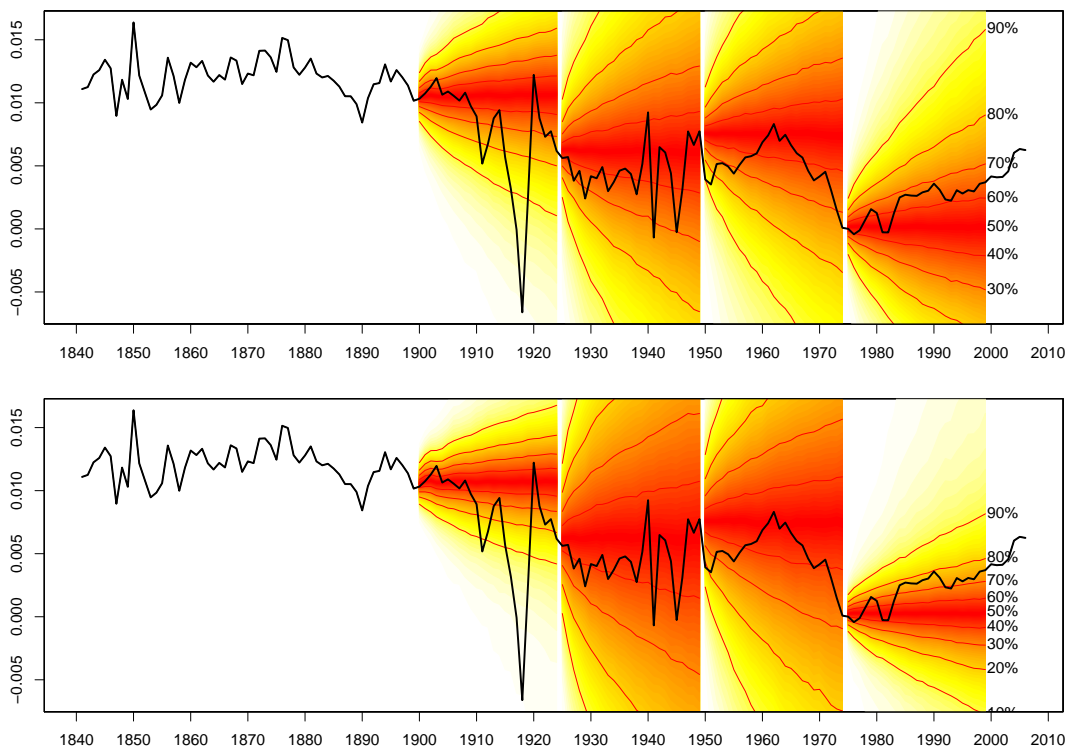


\section{Conclusion}

In this paper, we have explored the use of Bayesian models to forecast a single time series of population data in England and Wales from 1841 to 2007 forward to 2032. The key issue has been the specification of uncertainty in the forecasts. We have shown that both the forecasted medians and measures of uncertainly can differ considerably depending on the time series model used. This was reconciled by developing a Bayesian approach which combines forecasts from different models, with weights determined by how well models fit the observed data. We found that models which allow the underlying variance to shift at particular time points fitted the observed data well. They also provided a realistic assessment of forecast uncertainty which balances recent levels of variability with the probability that variance shifts may occur in the future, at a rate which is estimated based on the historical prevalence of such events.

The contributions of this paper are threefold. First, we have presented the Bayesian approach to time series forecasting for use in population applications. The computational steps required to estimate the model parameters have also been illustrated. Second, we have shown how Bayesian models are capable of including multiple sources of uncertainty. In particular, we extended standard autoregressive time series models to include stochastic volatility, random variance shifts and model averaging. In this paper, we have introduced these ideas by using relatively simple time series data, in order to develop a framework for incorporating Bayesian methods to produce population forecasts, and to gain a good understanding of the benefits. The results show the limitations of using a single model, particularly in the specification of uncertainty. While the underlying inputs we have used in this paper are relatively simple, the results can be used to provide a benchmark for specifying uncertainty in more complex projection situations that include, for example, age and demographic components of change (fertility, mortality and migration). These methods will also be valuable for forecasting other single demographic indicator variables, such as total fertility rates or life expectancies. Third, we have applied random variance shift models based on McCulloch and Tsay (1993), to forecast future population. We found these models, which to our knowledge have not previously been applied to demographic data, effectively controlled forecasts for the observed volatility.

Future work should consider modelling the demographic components of population change. Separate series of births, deaths and migration can be modelled as a multivariate time series process using Bayesian vector autoregressive models. This decomposition may be further continued by modelling subnational populations (and the flows between them) and by incorporating age structures. These extensions, which we are currently investigating, are likely to reduce the uncertainty of population forecasts in comparison to those presented in this paper. Here, the recent work on modelling fertility (Tuljapurkar 1999; Heilig et al. 2010; Alkema et al. 2011, 2012), mortality (Pedroza 2006; Girosi and 
King 2008; Raftery et al. 2013) and migration (Brierley et al. 2008; Bijak 2010; Raymer, J., Wiśniowski, A., Forster, J.J., Smith, P.W.F. and Bijak, J. 2013) provide good starting points. However, as the projection models become more complex, the relative importance of expert opinion will increase (see Bijak and Wiśniowski (2010) ). Fortunately, the Bayesian approach allows data and uncertainty in parameters and model choice to be fully quantified using probability distributions. In our implementation, prior distributions, which was kept weakly informative, had minimal influence on the final forecasts. This is not likely to be the case with more detailed forecasts, or in situations where the availability of data is lacking.

In conclusion, this work is relevant as most statistical agencies still rely on 'high' and 'low' variants to communicate uncertainty around their principal population projections. Such variants have a number of drawbacks with the most prominent being a lack of specificity regarding the probability range of the high, low or even principal variants (for discussion, see Keilman, Pham, and Hetland (2002) or Lutz and Goldstein (2004)). In response, demographers and statisticians have developed frequentist methods to calculate probabilistic forecasts that describe the uncertainly of future populations by relying on time series models, expert judgements or extrapolation of past forecast errors (Keilman 2001; Keilman, Pham, and Hetland 2002). Methods have also been developed to combine elements of each of these approaches, for example, the parameters from time series models have been constrained according to expert opinions (Lee and Tuljapurkar 1994) or to target levels and age distributions of fertility and mortality (Lutz, Sanderson, and Scherbov 2001). However, the use of Bayesian methods, which have the potential to bring all of these ideas together, are only recently gaining prominence in population forecasting (Bryant and Graham 2013; Raftery et al. 2012). We hope this paper illustrates some of the advantages of the Bayesian approach and motivates researchers to carefully consider not only if but how they include uncertainty in their forecasts.

\section{Acknowledgements}

This research is funded by ESRC Grant number RES-625-28-0001. The Centre for Population Change is a joint initiative between the University of Southampton and a consortium of Scottish Universities in partnership with the Office for National Statistics (ONS) and the General Register Office for Scotland (GROS). The findings, interpretations, and conclusions expressed in this paper are entirely those of the authors and should not be attributed in any manner to ONS or GROS. The authors would like to the thank the reviewers and Associated Editor for their comments and suggestions on an earlier version, Andrei Rogers for his advice concerning the direction of this paper and Arkadiusz Wiśniowski for his suggestions concerning the modelling. 


\section{References}

Abel, G.J. (2012). fanplot: Visualisations of sequential probability distributions. URL: http://cran.r-project.org/web/packages/fanplot/.

Abel, G.J. (2013). tsbugs: Create time series BUGS models. URL: http://cran.r-project.org/web/packages/tsbugs/.

Abel, G.J. and Wong, J.T.S. (2013). tsbridge: Calculate normalising constants for Bayesian time series models. URL: http://cran.r-project.org/web/packages/tsbridge/.

Ahlburg, D. (1987). Population Forecasts for South Pacific Nations using Autoregressive Models 1985-2000. Journal of Population Research 4(2): 157-167. doi:10.1007/BF03029414.

Alho, J.M. and Spencer, B.D. (2005). Statistical Demography and Forecasting. New York, USA: Springer.

Alho, J.M. and Spencer, B.D. (1985). Uncertain Population Forecasting. Journal of the American Statistical Association 80(390): 306-314. doi:10.2307/2287887.

Alkema, L., Raftery, A.E., Gerland, P., Clark, S.J., and Pelletier, F. (2012). Estimating trends in the total fertility rate with uncertainty using imperfect data: Examples from West Africa. Demographic Research 26(15): 331-362. doi:10.4054/DemRes.2012.26.15.

Alkema, L., Raftery, A.E., Gerland, P., Clark, S.J., Pelletier, F., Buettner, T., and Heilig, G.K. (2011). Probabilistic Projections of the Total Fertility Rate for All Countries. Demography 48(3): 815-839.

Anderson, M. (ed.) (1996). British Population History: From the Black Death to the Present Day. Cambridge, United Kingdom: Cambridge University Press.

Bijak, J. (2010). Forecasting International Migration in Europe: A Bayesian View. Dordrecht, Netherlands: Springer, 1st ed.

Bijak, J. and Wiśniowski, A. (2010). Bayesian forecasting of immigration to selected European countries by using expert knowledge. Journal of the Royal Statistical Society: Series A (Statistics in Society) 173(4): 775-796. doi:10.1111/ j.1467-985X.2009.00635.x.

Brierley, M.J., Forster, J.J., McDonald, J.W., and Smith, P.W.F. (2008). Bayesian Estimation of Migration Flows. In: Raymer, J. and Willekens, F. (eds.). International Migration in Europe. Chichester, United Kingdom: Wiley: 149-174.

Bryant, J.R. and Graham, P.J. (2013). Bayesian demographic accounts: Subnational 
Abel et al.: Integrating uncertainty in time series population forecasts

population estimation using multiple data sources. Bayesian Analysis 8(2): 1-34. doi:10.1214/13-BA820.

Chatfield, C. (2003). The Analysis of Time Series: An Introduction. Texts in Statistical Science. Boca Raton, USA: Chapman and Hall/CRC, 6th ed.

Coleman, D. and Salt, J. (1992). The British Population: Patterns, Trends, and Processes. Oxford, USA: Oxford University Press.

Gelman, A., Carlin, J.B., Stern, H.S., and Rubin, D.B. (2003). Bayesian Data Analysis. Boca Raton, USA: Chapman \& Hall/CRC, 2nd ed.

Girosi, F. and King, G. (2008). Demographic Forecasting. Princeton, USA: Princeton University Press.

Heilig, G., Buettner, T., Li, N., Gerland, P., Alkema, L., Chunn, J., and Raftery, A. (2010). A stochastic version of the United Nations World Population Prospects: Methodological improvements by using Bayesian fertility and mortality projections. Lisbon, Portugal: Joint Eurostat/UNECE Work Session on Demographic Projections. URL: www.unece.org/fileadmin/DAM/stats/documents/ece/ces/ge.11/2010/wp.14.e.pdf.

Hinde, A. (2003). England's Population: A History since the Domesday Survey. London, United Kingdom: Arnold Publication.

Hoeting, J.A., Madigan, D., Raftery, A.E., and Volinsky, C.T. (1999). Bayesian Model Averaging: A Tutorial. Statistical Science 14(4): 382-401. doi:10.2307/2676803.

Keilman, N. (2001). Demography. Uncertain population forecasts. Nature 412(6846): 490-491. doi:10.1038/35087685.

Keilman, N. and Pham, D.Q. (2004). Time Series Based Errors and Empirical Errors in Fertility Forecasts in the Nordic Countries. International Statistical Review/Revue Internationale de Statistique 72(1): 5-18. doi:10.2307/1403839.

Keilman, N., Pham, D.Q., and Hetland, A. (2002). Why population forecasts should be probabilistic - illustrated by the case of Norway. Demographic Research 6: 409-454. doi:10.4054/DemRes.2002.6.15.

Keyfitz, N. (1991). Uncertainty in National Population Forecasting: Issues, Backgrounds, Analyses, Recommendations. Population Studies: A Journal of Demography 45(3): 545-546. doi:10.1080/0032472031000145786.

Lee, R.D. (1998). Probabilistic Approaches to Population Forecasting. Population and Development Review 24: 156-190. doi:10.2307/2808055.

Lee, R.D. and Tuljapurkar, S. (1994). Stochastic Population Forecasts for the United 
States: Beyond High, Medium, and Low. Journal of the American Statistical Association 89(428): 1175-1189. doi:10.2307/2290980.

Lunn, D., Spiegelhalter, D., Thomas, A., and Best, N. (2009). The BUGS project: Evolution, critique and future directions. Statistics in Medicine 28(25): 3049-3067. doi:10.1002/sim.3680.

Lutz, W. and Goldstein, J.R. (2004). Introduction: How to Deal with Uncertainty in Population Forecasting? International Statistical Review/Revue Internationale de Statistique 72(1): 1-4. doi:10.1111/j.1751-5823.2004.tb00219.x.

Lutz, W., Sanderson, W., and Scherbov, S. (2001). The end of world population growth. Nature 412(6846): 543-545.

McCulloch, R.E. and Tsay, R.S. (1993). Bayesian Inference and Prediction for Mean and Variance Shifts in Autoregressive Time Series. Journal of the American Statistical Association 88(423): 968-978. doi:10.1080/01621459.1993.10476364.

Meng, X.L. and Wong, W.H. (1996). Simulating Ratios of Normalizing Constants via a Simple Identity: A Theoretical Exploration. Statistica Sinica 6: 831-860. URL: http://www3.stat.sinica.edu.tw/statistica/oldpdf/A6n43.pdf.

O'Hagan, A. and Forster, J.J. (2004). Bayesian Inference. Kendalls Advanced Theory of Statistics. London, United Kingdom: Arnold Publication, 2nd ed.

Pedroza, C. (2006). A Bayesian forecasting model: predicting U.S. male mortality. Biostatistics 7(4): 530-550. doi:10.1093/biostatistics/kxj024.

Pflaumer, P. (1992). Forecasting US population totals with the Box-Jenkins approach. International Journal of Forecasting 8(3): 329-338. doi:10.1016/ 0169-2070(92)90051-A.

Plummer, M., Best, N., Cowles, K., and Vines, K. (2006). CODA: Convergence diagnosis and output analysis for MCMC. R News 6(1): 7-11. URL: http://cran.r-project.org/doc/Rnews/Rnews_2006-1.pdf.

Raftery, A.E., Chunn, J.L., Gerland, P., and Sevčíková, H. (2013). Bayesian probabilistic projections of life expectancy for all countries. Demography 50(3): 777-801.

Raftery, A.E., Li, N., Ševčíková, H., Gerland, P., and Heilig, G.K. (2012). Bayesian probabilistic population projections for all countries. Proceedings of the $\mathrm{Na}$ tional Academy of Sciences of the United States of America 109(35): 13915-21. doi:10.1073/pnas.1211452109.

Raymer, J., Forster, J.J., Smith, P.W.F., Bijak, J., and Wiśniowski, A. (2012). Integrated 
Modelling of European Migration: Background, Specification and Results. NORFACE Migration Discussion Paper (4).

Raymer, J., Wiśniowski, A., Forster, J.J., Smith, P.W.F. and Bijak, J. (2013). Integrated Modeling of European Migration. Journal of the American Statistical Association 108(503): 801-819. doi:10.1080/01621459.2013.789435.

Saboia, J.L.M. (1974). Modeling and Forecasting Populations by Time Series: The Swedish Case. Demography 11(3): 483-492. doi:10.2307/2060440.

Sturtz, S., Ligges, U., and Gelman, A. (2005). R2WinBUGS: a package for running WinBUGS from R. Journal of Statistical Software 12(3): 1-16.

Tayman, J., Smith, S.K., and Lin, J. (2007). Precision, bias, and uncertainty for state population forecasts: an exploratory analysis of time series models. Population Research and Policy Review 26(3): 347-369. doi:10.1007/s11113-007-9034-9.

Tuljapurkar, S. (1999). Validation, probability-weighted priors, and information in stochastic forecasts. International Journal of Forecasting 15(3): 259-271. doi:10.1016/S0169-2070(98)00082-X.

Wrigley, E.A. and Schofield, R.S. (1989). The Population History of England 15411871. Cambridge Studies in Population, Economy and Society in Past Time. Cambridge, United Kingdom: Cambridge University Press, 1st ed. 


\section{Appendix}

Adding a plot of prior distributions to those generated from the plot.memc command in the coda package is not straight forward. In this appendix we illustrate some R code to enable the prior and posterior distributions to appear on the same plot for each of the model types illustrated in this paper.

The nodes function in the tsbugs package provides a data. frame on the parameters used in the prior part of the BUGS script. Using the ar2 object (see Section 4.1) we may illustrates its output,

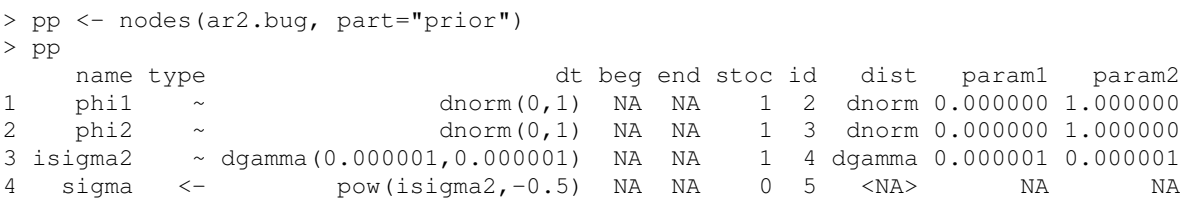

The MCMC simulations for these parameters, stored in the ar2 object can be extracted after defining our parameter set, thet a, as those parameters that have prior distributions directly defined in the BUGS script,

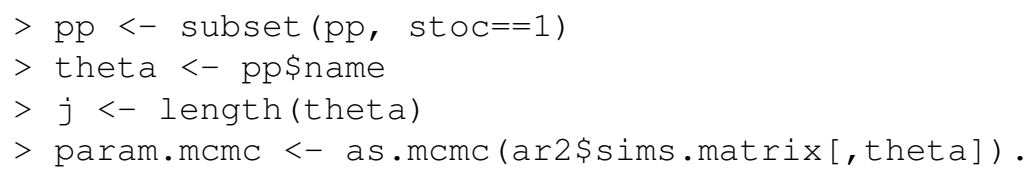

These simulations, that are now mcmc type objects, can be plotted using the plot. mcmc command,



Note, we suppress the auto. layout in the plot.mcmc, allowing us to fix the plotting area to take $j$ rows (the number of parameters) and two columns defined in the previous line. This produces a plot, much like that in Figure 2 in the paper. 
To add the posterior densities to the plots, we consider each density plot in the second column within the for loop below,

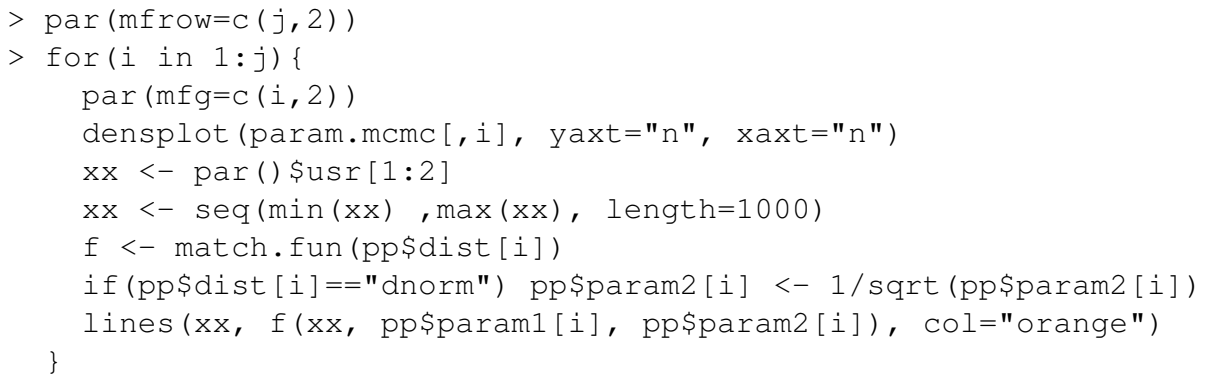

Within the loop, we first select the plotting area using the mfg graphical parameter, then overlay the exiting plot with the same posterior density. Second, using the us $r$ graphical parameter, information on the range of the $\mathrm{x}$-axis is first saved, and then used to create a sequence of numbers within the minimum and maximum values. Third, the distribution function of the prior parameter is stored as function labelled $\mathrm{f}$. In addition, if this function is dnorm the second, precision parameter, stored in the pp object is converted to a standard deviation. Finally, the density of the given prior distributions and its parameters are calculated for values over the range of the $\mathrm{x}$-axis and plotted as a orange line. The output from this sequence of code for the ar 2 object is shown in Figure A-1.

The code above can also be used to plot prior and posterior distributions for the stochastic volatility and random variance shifts models. This can be done after defining $\mathrm{pp}$ as the BUGS script produced in the paper, from sv2 . bug or rv2 . bug and the param. mcmc object as the corresponding MCMC simulation results, stored in $\mathrm{SV} 2$ or rv2. These plots are shown in Figures A-2 and A-3. 
Figure A-1: $\quad$ Trace plots, posterior and prior (orange) densities of AR(2) model parameters
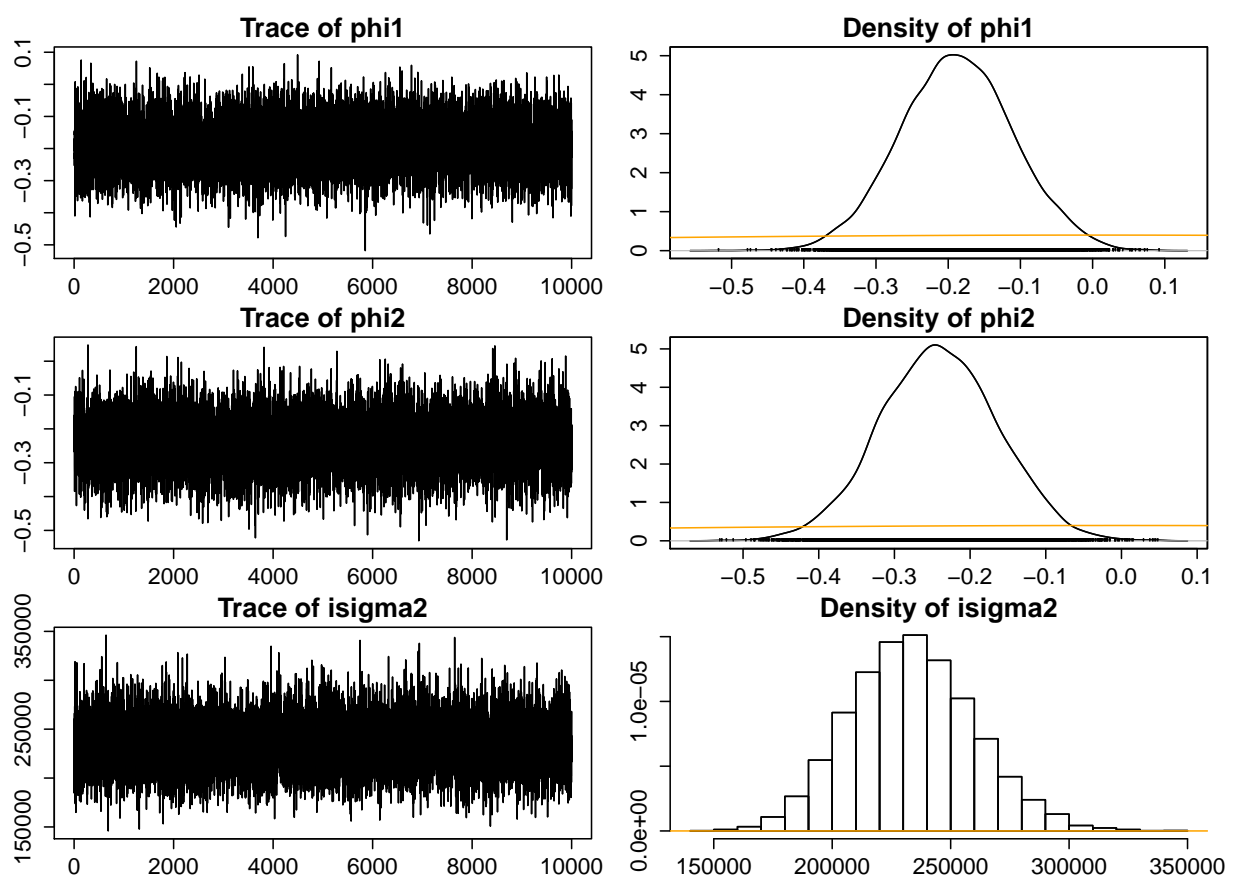
Abel et al.: Integrating uncertainty in time series population forecasts

Figure A-2: $\quad$ Trace plots, posterior and prior (orange) densities of AR(2)-SV model parameters
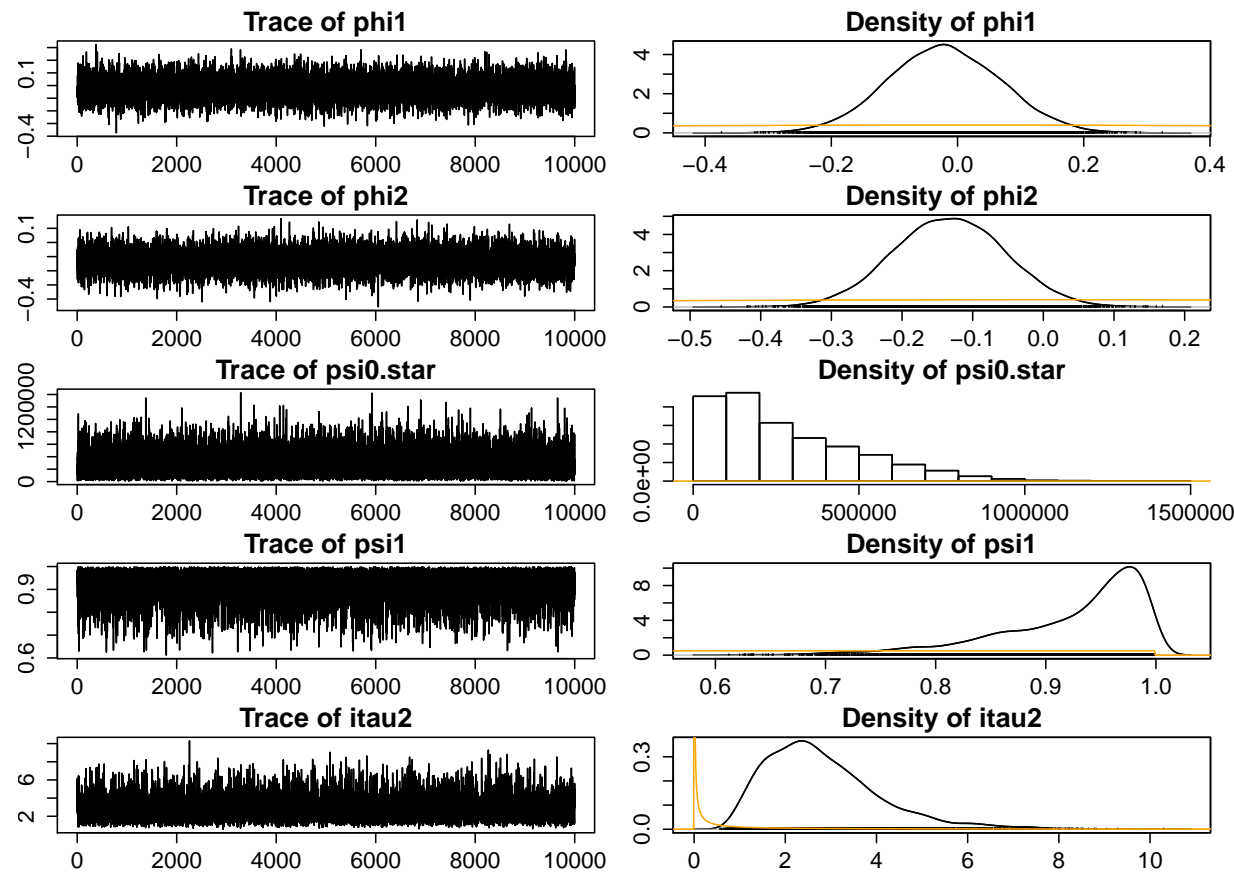
Figure A-3: $\quad$ Trace plots, posterior and prior (orange) densities of AR(2)-RV model parameters
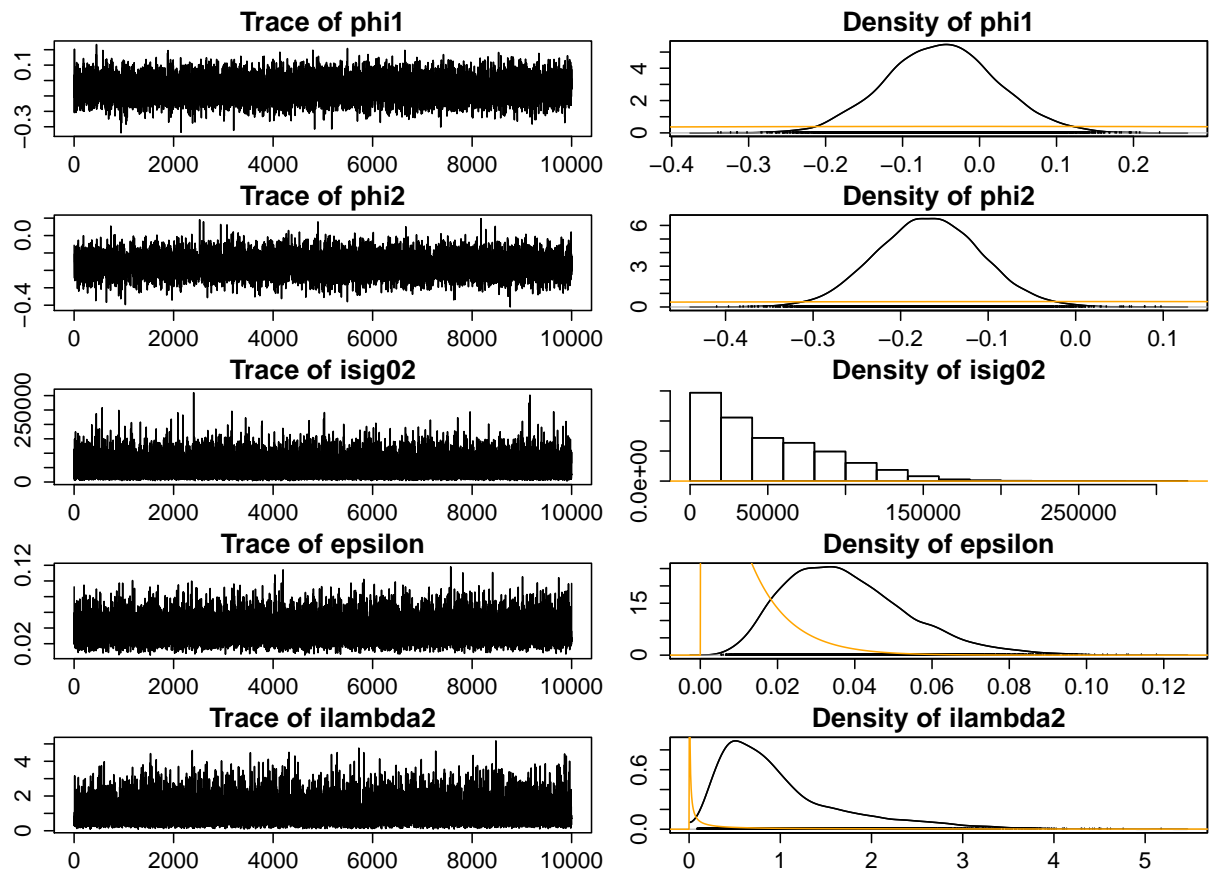
Abel et al.: Integrating uncertainty in time series population forecasts 